\title{
ADMISIÓN UNIVERSITARIA: EL CASO DEL PUNTAJE RANKING Y LA RETENCIÓN DE LOS BENEFICIADOS
}

\section{Andrés Barrios F.}

London School of Economics

\begin{abstract}
Resumen: En 2012, el Consejo de Rectores de las Universidades Chilenas decidió incorporar el puntaje ranking en el proceso de admisión que tiene a su cargo. Con este cambio, buscaba reconocer el mérito académico de los estudiantes en su contexto escolar y, a través de ello, reducir la desigualdad en el acceso a las universidades sin sacrificar la calidad de los seleccionados. Esta investigación estudia las tasas de retención de los estudiantes beneficiados por esta política, tras su primer año en el sistema. Para identificar al grupo de estudiantes beneficiados se simularon los resultados del proceso de admisión bajo distintos escenarios. Se encontró que, pese a que las tasas de retención a nivel de sistema, de institución y de programa
\end{abstract}

\footnotetext{
Andrés Barrios F. Economista de la Pontificia Universidad Católica. Estudiante de la London School of Economics. Email: m.barrios-fernandez@1se.ac.uk.

El autor agradece a Steve Pischke y a Esteban Aucejo por sus múltiples consejos y comentarios, y también a Sebastián Kreft por su ayuda en la implementación del algoritmo de selección. También quiere agradecer tanto al SIES y al Centro de Estudios del Ministerio de Educación como al Demre, por darle acceso a los datos administrativos que usó en este trabajo. Agradece, además, al Centro de Estudios Públicos y en especial a Harald Beyer y a Loreto Cox, por el apoyo dado en este proyecto. Por último, aprecia los comentarios realizados por los árbitros anónimos de Estudios Públicos. Los potenciales errores presentes en este documento son de responsabilidad exclusiva del autor.
} 
no son necesariamente bajas, los beneficiados por el puntaje ranking muestran menores tasas que sus compañeros, que el 10 por ciento inferior de los seleccionados y que los estudiantes que fueron perjudicados por esta política.

PALABRAS ClAVE: puntaje ranking, equidad, proceso de selección universitaria.

RECIBIDO: enero 2018; ACEPTADO: marzo 2018.

\section{UNIVERSITY ADMISSION: THE RANKING SCORE SYSTEM AND BENEFICIARY RETENTION}

ABSTRACT: In 2012, the Council of Chilean University Rectors decided to incorporate the so-called ranking score, which rates university applicants relative to the overall performance of the school they have been attending, into the admissions process it manages. This change was designed to recognize students' academic merit in the context of their school and thus reduce inequality in university access without lowering quality. The present study looks at the retention rates of students benefiting from this policy after their first year in the system. To identify the beneficiary group, admission process outcomes were simulated under different scenarios. The finding is that while retention rates at the system, institution and course level are not necessarily low, ranking score beneficiaries evince lower rates than their fellow students, than the bottom 10 per cent of successful applicants and than students who have lost out by this policy.

KEYWORDS: ranking score, equity, university selection process.

RECEIVED: January 2018; ACCEPTED: March 2018.

\section{INTRODUCCIÓN}

a búsqueda de un acceso equitativo a la educación superior es un desafío que comparten tantos países desarrollados, como en vías de desarrollo. Lograr mayores niveles de equidad en este nivel educativo puede contribuir al crecimiento económico (Oded 2011) y al fortalecimiento de la democracia (Lance 2011). Además, puede ser en muchos casos deseable desde una perspectiva de justicia social.

Chile no es una excepción a esta tendencia y, de hecho, existen múltiples iniciativas, tanto públicas como privadas, que han buscado 
avanzar en esta dirección. ${ }^{1}$ Una de ellas es la introducción del puntaje ranking en el sistema de selección universitario a cargo del Consejo de Rectores de las Universidades Chilenas (Cruch).

Esta medida - inspirada en los "percentage admission plans", implementados en Estados Unidos — 2 buscaba aumentar la equidad en el acceso a programas universitarios, sin sacrificar la calidad de los seleccionados en términos de su rendimiento académico.

En una investigación complementaria (Barrios 2017) se estudia los efectos de este instrumento en términos de equidad en el acceso. De acuerdo a los resultados arribados, los cambios observados en el sistema y en el subconjunto de universidades altamente selectivas generaron ganancias modestas en equidad. ${ }^{3}$ Estos resultados son consistentes con lo encontrado por Larrocau et al. (2013), quienes documentan que menos del 1 por ciento de los postulantes ingresó al sistema gracias al puntaje ranking en su primer año de funcionamiento. En una línea similar, Santalices et al. (2017) sostienen que los efectos de estos cambios en términos de equidad fueron pequeños.

En esta investigación, estudio las tasas de retención al primer año de los estudiantes beneficiados por esta política. Ésta es la única medida de desempeño que observo en la totalidad de las instituciones que participan del Sistema Único de Admisión, y aunque sería interesante contar con otros indicadores, como avance curricular y promedios de notas, las dificultades para acceder a este tipo de información para la totalidad de

${ }^{1}$ Algunos ejemplos incluyen el programa Propedéutico Unesco, la Beca de Excelencia Académica y los cupos supernumerarios, además de iniciativas como Talento e Inclusión, impulsadas por la Universidad Católica, y los cupos de equidad de la Universidad de Chile. El programa PACE es otro ejemplo de este tipo de iniciativas.

${ }^{2}$ Los "percentage admission plans" fueron implementados en estados como California, Texas y Florida, tras una serie de fallos de la Corte Suprema que prohibieron el uso de políticas de discriminación positiva en los procesos de admisión universitaria. Estos programas buscan aumentar la participación de grupos subrepresentados, ofreciendo acceso directo a la universidad a estudiantes que finalizan sus estudios secundarios en el top 5 por ciento - top 20 por ciento de su generación.

${ }^{3}$ Los resultados de Barrios (2017) muestran que, con la inclusión del ranking, aumenta la diferencia en las probabilidades de admisión entre estudiantes destacados y no destacados en términos de las notas de enseñanza media. Sin embargo, encuentra que estas diferencias no se traducen en una mayor equidad; no se observan cambios importantes en las probabilidades de admisión al sistema y a instituciones altamente selectivas para estudiantes provenientes de contextos más desaventajados. 
las instituciones me obligan a concentrarme únicamente en esta variable (tasa de retención al primer año). ${ }^{4}$ Cabe destacar que para que una mayor equidad en el acceso se traduzca en mayor integración y movilidad social, es fundamental conocer lo que ocurre con los estudiantes una vez que son admitidos en la universidad. No tendría mayor sentido implementar una política en la que se incrementara la equidad en el acceso pero no en la permanencia y titulación de los estudiantes.

La evidencia sobre los efectos de este tipo de medidas en la calidad del pool de seleccionados es mixta. Antonovics y Backes (2014) investigan lo que ocurrió en el sistema de admisión de la Universidad de California tras la prohibición del uso de acción afirmativa basada en la raza de los postulantes. Encuentran que, como respuesta a esta prohibición, la universidad modificó los pesos dados al SAT y al rendimiento escolar de los estudiantes en su proceso de admisión, logrando con ello contrarrestar parcialmente la caída en la participación de minorías en sus instituciones. Encuentran además que la calidad de los estudiantes, medida en términos de su promedio ponderado al final del primer año, no se ve afectada por los cambios implementados por la universidad. Kapor (2016) estudia el programa Texas Top Ten y encuentra que éste aumentó la participación de minorías en las universidades emblemáticas del Estado; sostiene además que este programa permite seleccionar a mejores postulantes que un sistema que premiara directamente a estudiantes provenientes de estas minorías. Arcidiacono et al. (2014) estudian lo que ocurre con las tasas de graduación de minorías raciales tras la prohibición del uso de acción afirmativa en la Universidad de California. Encuentran que las tasas de graduación de estos grupos aumen-

\footnotetext{
${ }^{4}$ La deserción es un fenómeno con múltiples causas, que incluyen el potencial académico, pero también otras variables como características socioeconómicas, habilidades sociales y la capacidad de adaptación de los estudiantes. Desde esta perspectiva, utilizar las tasas de retención como una medida de potencial académico no es totalmente correcto; estudiantes con potenciales académicos muy altos podrían abandonar un programa o institución debido a alguna de las otras razones mencionadas. Cabe destacar, en todo caso, que los otros indicadores de rendimiento académico que pudiesen utilizarse también son afectados por estas medidas. De todas formas, resulta interesante estudiar las tasas de retención de los beneficiados. Si estos estudiantes presentan tasas de retención menores a las de sus compañeros y a las de quienes hubiesen ingresado en su lugar, es importante documentar estas diferencias para pensar en las medidas que pudiesen tomarse para asegurarse de que las modestas mejoras en equidad en el acceso documentadas por Barrios (2017) se traduzcan también en mayores niveles de equidad en la titulación.
} 
tan, en parte porque estos estudiantes se mueven a campus menos selectivos, mejorando la calidad del match entre estudiante e institución. De acuerdo a estos resultados, el mejorar el acceso de grupos subrepresentados a instituciones altamente selectivas no es necesariamente algo que los beneficie. Cortes (2010) estudia la situación de los estudiantes provenientes de grupos desaventajados tras el fin de los programas de acción afirmativa y la implementación de los percentage admission plans, y encuentra que tras esta inclusión las tasas de retención y graduación de estos grupos disminuyeron.

En una línea más cercana a este trabajo, Arcidiacono et al. (2012) investigan cómo evoluciona la diferencia en las notas de estudiantes blancos y de los pertenecientes a minorías raciales que fueron admitidos a través de programas de acción afirmativa. Sus resultados muestran que la aparente convergencia en rendimientos esconde cambios en los tipos de programas y cursos tomados por estudiantes provenientes de distintos contextos.

En el contexto chileno, Koljatic y Silva (2013) estudian el programa propedéutico de la Universidad de Santiago. ${ }^{5}$ Encuentran que los estudiantes admitidos a través de este programa presentan tasas de retención más bajas que las de sus pares admitidos a través de la vía regular, pero que su rendimiento académico mejora en el tiempo. Sin embargo, tras tomar en cuenta los altos niveles de vulnerabilidad de los participantes de este programa, evalúan sus resultados como esperanzadores. El Consejo de Rectores de las Universidades Chilenas (Cruch 2017) estudió el poder predictivo del puntaje de ranking en el rendimiento académico de los estudiantes. En este estudio encuentran que, aunque contribuye a explicar parte del promedio ponderado de los estudiantes, su aporte es bastante menor en comparación con el de los otros instrumentos de selección (PSU y notas de enseñanza media). ${ }^{6}$

${ }^{5}$ El programa propedéutico ofrece acceso a la universidad a estudiantes destacados provenientes de contextos vulnerables. Estudiantes en el top 10 por ciento de su generación son acompañados durante su último año de educación secundaria, tras lo que se les ofrece acceso a un programa de bachillerato en el que continúan recibiendo apoyo para integrarse adecuadamente a la universidad.

${ }^{6}$ Por su naturaleza, esta investigación trabaja exclusivamente con estudiantes matriculados en las universidades. Es decir, son alumnos que obtuvieron notas y puntajes PSU lo suficientemente altos como para ser admitidos en estas instituciones. Esto nos obliga a ser cautelosos a la hora de interpretar estos resultados, ya que éstos no necesariamente reflejan el poder predictivo de los distintos instrumentos estudiados en otros rangos de puntaje. 
Gil et al. (2013), por otro lado, comparan el rendimiento de estudiantes de la Universidad Católica que egresaron de enseñanza media en el top 10 por ciento de su establecimiento, con quienes obtuvieron un rendimiento escolar menor. Encuentran que, una vez en la universidad, estos alumnos presentan tasas de retención y promedios ponderados más altos. Extienden este análisis a estudiantes que hubiesen obtenido un puntaje de ranking en el top 10 por ciento, encontrando resultados similares. ${ }^{7}$ A partir de estos resultados, validan el uso de este instrumento de selección en el sistema. ${ }^{8}$

Santalices et al. (2017) estudian el poder predictivo del ranking de egreso de enseñanza media en las tasas de permanencia, tanto a nivel de sistema como a nivel institucional. En su análisis aplican tres definiciones de ranking: (1) puntaje de ranking utilizado en el sistema único de admisión (ranking-bono-SUA), (2) percentil de egreso y (3) quintil de egreso. Sus resultados muestran que, tras controlar por el rendimiento de los estudiantes en el resto de los instrumentos de selección (PSU, NEM), los tres indicadores de ranking contribuyen a predecir la retención institucional sólo en las universidades del Cruch. ${ }^{9}$ Cabe destacar que los autores nos llaman a interpretar estos resultados con cautela y advierten que no son causales. Adicionalmente, San-

${ }^{7}$ No resulta del todo claro la forma en que construyen esta última medida; por la manera en que está descrita, pudiesen ser estudiantes sobre el percentil 90 de la distribución nacional del puntaje ranking o sobre el percentil 90 al interior del establecimiento escolar de egreso. Evidentemente, ambas definiciones no son equivalentes y pudiesen llevarnos a conclusiones diferentes.

${ }^{8}$ Es importante ser cautelosos a la hora de interpretar estos resultados. En primer lugar, los estudiantes de la Universidad Católica no son necesariamente representativos del resto de los estudiantes del sistema. Puede haber diferencias tanto en características académicas como socioeconómicas que hagan difícil extender estos resultados a otros contextos. Además, las comparaciones presentadas en este estudio no controlan por los resultados de los estudiantes en la PSU. En esta muestra, estudiantes en el top 10 por ciento podrían ser también quienes obtienen mayores puntajes en la PSU. De ser éste el caso, la interpretación de los resultados no sería correcta. Al trabajar exclusivamente con seleccionados de esta universidad, se controla hasta cierto punto por esta variable. Sin embargo, incluso dentro de esta institución hay diferencias importantes en el rendimiento de los estudiantes en las distintas secciones de la PSU.

${ }^{9}$ Este análisis se concentra en los procesos 2007-2011; es decir, el período previo a la incorporación del puntaje ranking. Encuentran que este último no contribuye a predecir retención a nivel de sistema, ni a nivel institucional en instituciones no Cruch. 
talices et al. (2017) encuentran que, al controlar por características socioeconómicas de los estudiantes, el poder predictivo del puntaje de ranking (ranking-bono-SUA) desaparece. Esto es consistente con la discusión presentada en Barrios (2017), en que se muestra que el puntaje de ranking es mayor para estudiantes provenientes de contextos más aventajados; su poder predictivo, por lo tanto, se explicaría principalmente por su relación con las características socioeconómicas de los estudiantes.

En este trabajo, a diferencia de los estudios realizados por el Cruch (2017) y Santalices et al. (2017), el foco está en las tasas de retención de los estudiantes que fueron beneficiados y perjudicados por el puntaje ranking. Estudiaré lo que ocurre en la totalidad de las instituciones que participan del sistema único de admisión. Con este análisis, busco superar además algunos de los desafíos metodológicos de Gil et al. (2013). ${ }^{10}$

Para identificar al grupo de estudiantes beneficiados y perjudicados, aprovecho la disponibilidad de ricos datos administrativos y simulo los resultados del proceso de admisión bajo distintos escenarios. A partir de estas simulaciones, defino dos categorías de beneficiados. La primera incluye a aquellos estudiantes que sin el puntaje ranking no hubiesen sido admitidos en ninguna de las instituciones que participan del sistema único de admisión, pero que, gracias a su inclusión, pudieron acceder a ellas. La segunda categoría incluye a aquellos estudiantes que gracias al puntaje ranking ingresaron a preferencias más altas dentro de su lista de postulaciones. De manera análoga, defino también a dos grupos de perjudicados.

Al estudiar las tasas de retención del primer grupo de estudiantes beneficiados, encuentro que éstas son entre 7 y 8 puntos porcentuales menores que las de sus compañeros en el sistema, ${ }^{11}$ entre 5 y 6 puntos

${ }^{10}$ Como se discute en la nota al pie anterior, existen dudas tanto sobre la validez externa como interna del análisis de Gil et al. (2013). Una de las dificultades que presenta, es que en ninguno de sus análisis controla por el puntaje obtenido por los estudiantes en la PSU. Es difícil creer que esta variable no esté correlacionada con el rendimiento escolar y el rendimiento universitario. De ser éste el caso, sus comparaciones estarían sesgadas.

${ }^{11}$ El sistema se refiere a la totalidad de instituciones que participan del sistema único de admisión. A menos que se especifique lo contrario, los análisis presentados en esta investigación trabajan con este universo de instituciones. 
porcentuales menores que las del 10 por ciento inferior de los seleccionados a cada programa ${ }^{12}$ y entre 2 y 3 puntos porcentuales menores que las del grupo de estudiantes perjudicados. Esta última diferencia equivale a una tasa de deserción 23,2 por ciento mayor a nivel de sistema, 13,3 por ciento mayor a nivel institucional y 9,8 por ciento mayor a nivel de programa. La magnitud de las diferencias medidas en puntos porcentuales se mantiene en un rango similar al restringir el análisis exclusivamente a instituciones altamente selectivas.

Los análisis para el segundo grupo de beneficiados, es decir, aquellos estudiantes que gracias al puntaje ranking lograron ingresar a preferencias más altas, arrojan resultados similares. Una vez más, encuentro que sus tasas de retención son menores que las de sus compañeros, que las del 10 por ciento de seleccionados con menores puntajes y que las del grupo de estudiantes perjudicados (estudiantes que, en la ausencia del puntaje ranking, hubiesen sido admitidos en preferencias más altas). Las diferencias en relación con las tasas de deserción se encuentran en un rango similar a las descritas para la primera categoría de beneficiados.

A pesar de encontrar que las tasas de retención de los beneficiados son menores que las observadas para los perjudicados, es importante destacar que esto no significa necesariamente que ellas sean bajas. Bajo todas las definiciones de retención encuentro que estas tasas superan el 70 por ciento.

Es importante ser cuidadosos con la interpretación de estos resultados; la diferencia en las tasas de retención pudiese explicarse por diferencias en el potencial académico de los estudiantes, pero también por otros factores, como, por ejemplo, su capacidad para integrarse y adaptarse al sistema universitario. Probablemente, el resultado observado se debe a una mezcla de distintos factores, pero es relevante que las instituciones tengan en cuenta las diferencias en retención para que cambios al sistema de admisión vayan acompañados de programas de

12 Estos estudiantes corresponden a individuos que fueron admitidos a través de la vía regular a programas ofrecidos por las instituciones que participan del sistema único de admisión. Es decir, son alumnos que fueron admitidos junto a los estudiantes beneficiados, pero que ingresaron en el 10 por ciento inferior de su programa. 
apoyo a estudiantes que pudiesen enfrentar mayores dificultades una vez admitidos en el sistema. ${ }^{13}$

Este documento está organizado en cinco secciones. Luego de esta introducción, en la siguiente sección describo el sistema de educación superior chileno y el funcionamiento del puntaje ranking; luego describo los datos y la estrategia empírica utilizada; en la cuarta sección presento los resultados, y, finalmente, en la quinta sección, concluyo.

\section{SISTEMA UNIVERSITARIO Y EL PUNTAJE RANKING}

En Chile existen cerca de 60 universidades, 25 de las cuales forman parte del Consejo de Rectores de las Universidades Chilenas (Cruch). Las universidades del Cruch, y desde el año 2011 otras ocho universidades privadas, seleccionan a sus estudiantes utilizando un sistema centralizado de admisión. ${ }^{14}$ Para poder participar de este proceso, los postulantes deben completar su educación media y rendir la Prueba de Selección Universitaria (PSU).

Las instituciones definen el número de vacantes en cada uno de sus programas y deciden también los pesos que tendrán las distintas secciones de la PSU — lenguaje, matemáticas, ciencias y ciencias sociales-, así como las notas de enseñanza media al momento de asignar sus vacantes. Los potenciales postulantes conocen esta información antes de rendir la PSU y, por lo tanto, son variables que pueden considerar al elegir las instituciones y programas a los que postularán.

${ }^{13}$ Es importante mencionar que la existencia de múltiples factores que afectan la retención de estudiantes también aplica a otros indicadores de rendimiento académico. Las notas y el avance curricular de un estudiante, por ejemplo, no son consecuencia únicamente del potencial académico del mismo, sino que también de otros factores como los discutidos para el caso de la retención. Por ello, al medir la calidad de los estudiantes a partir de variables de desempeño en la universidad, se está midiendo un indicador que, además de su potencial académico, evalúa otras características de los estudiantes que explican su capacidad de adaptarse a la experiencia universitaria que les toca experimentar. Como esta experiencia depende también de características institucionales, el rendimiento académico de los estudiantes puede también afectarse a través de políticas institucionales.

${ }^{14}$ En 2011 se integraron ocho universidades y a partir de ese entonces otras instituciones privadas se han unido al sistema único de admisión. En el proceso de admisión 2018, además de las universidades del Cruch, participaron doce universidades privadas. 
Una vez recibidos sus resultados en la PSU, los estudiantes postulan a un máximo de diez preferencias a través de una plataforma online. Las vacantes se definen a través de un algoritmo de la familia GaleShapley, que asigna a cada estudiante a la preferencia más alta a la que sus puntajes le permiten acceder. Una vez que un estudiante es asignado a un programa, el resto de sus postulaciones son desechadas (independientemente de si sus puntajes le hubiesen permitido ser admitido a ellas).

Por otro lado, las universidades que no participan de este sistema centralizado de admisión seleccionan a sus estudiantes a través de procesos independientes. Sin embargo, para ellas la PSU sigue siendo relevante, en parte por la información que les entrega y, en parte, debido a la existencia de incentivos financieros vinculados a este instrumento.

En junio del año 2012, el Cruch anunció que sumaría un nuevo instrumento de selección en el proceso de admisión a las universidades que tendría lugar a finales de ese año: el puntaje ranking. Con su inclusión se pretendía aumentar la equidad en el acceso a las universidades, premiando el mérito académico de los estudiantes en su contexto escolar.

Esta modificación al sistema de admisión buscaba reconocer las diferencias existentes en las oportunidades de aprendizaje a las que tienen acceso jóvenes provenientes de distintos contextos, premiando el buen desempeño académico de los estudiantes en relación con otros jóvenes similares a ellos.

Con este fin, la fórmula escogida para el cálculo del puntaje ranking consideró, además de las notas del estudiante, el promedio y el máximo de las notas de enseñanza media de las cohortes egresadas del mismo establecimiento escolar durante los tres años anteriores. A partir de estos parámetros, se generó un puntaje que intentó tomar en cuenta la realidad de los estudiantes a lo largo de su etapa escolar.

El puntaje ranking se definió entonces como el puntaje asociado a las notas de enseñanza media (es decir, el puntaje NEM que ya era parte de los instrumentos de selección) y más un bono entregado a estudiantes cuyas notas de enseñanza media son superiores al promedio de las tres generaciones anteriores.

La figura 1 ilustra el funcionamiento de la fórmula de cálculo de este puntaje en mayor detalle. Como puede apreciarse, el puntaje ranking coincide con el puntaje NEM para todos aquellos estudiantes cuyo 
promedio de enseñanza media es inferior o igual al promedio de las tres generaciones anteriores. Estudiantes cuyo promedio de enseñanza media es mayor que el de las generaciones anteriores reciben un bono, por lo que su puntaje ranking es superior al puntaje NEM. Este bono aumenta hasta que se alcanza el promedio del máximo promedio de las tres generaciones anteriores; desde ese punto en adelante, el puntaje ranking permanece constante en 850 puntos (ver Barrios (2017) para un análisis más detallado de esta fórmula).

Figura 1. DISTRIBUCIÓN DEL PUNTAJE PROMEDIO EN LA PSU (2008-2015)

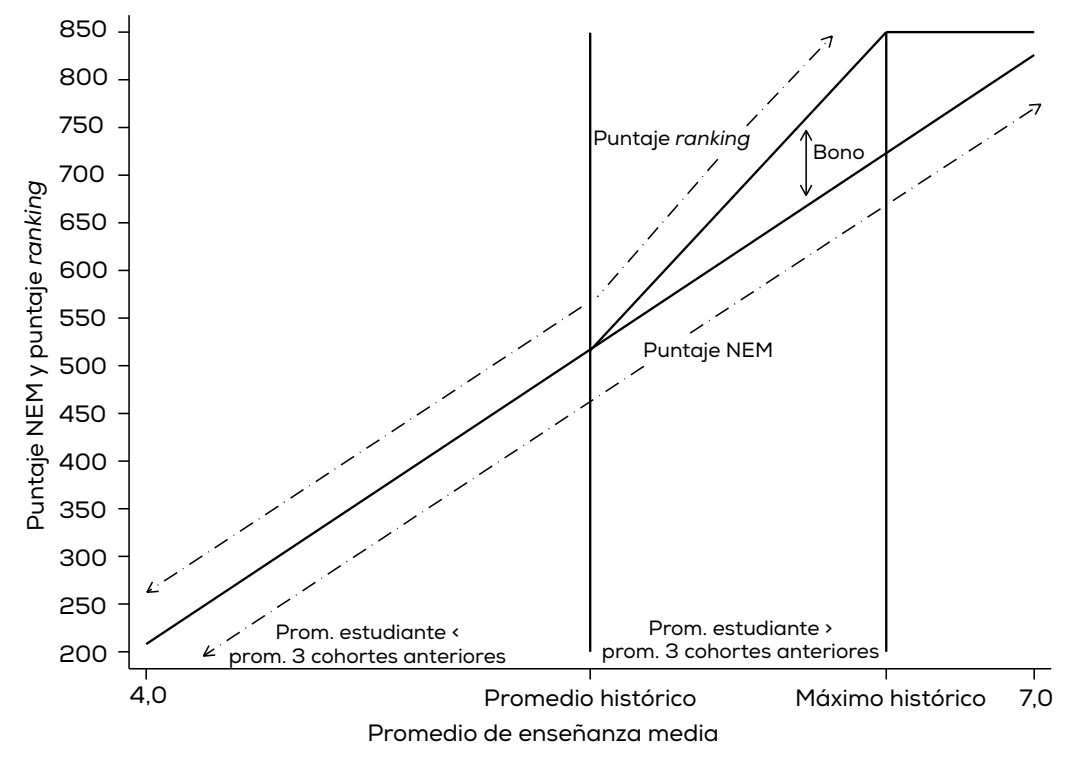

La figura ilustra el funcionamiento de la fórmula de cálculo del puntaje ranking. El puntaje ranking coincide con el puntaje NEM para todos aquellos estudiantes cuyo promedio de enseñanza media es inferior al promedio de enseñanza media de las tres generaciones anteriores. Estudiantes, cuyo promedio de enseñanza media es mayor que el de las tres generaciones anteriores, reciben un bono, por lo que su puntaje ranking es superior al puntaje NEM. Este bono aumenta hasta que se alcanza el promedio del máximo promedio de las tres generaciones anteriores; desde ese punto en adelante, el puntaje ranking permanece constante en 850 puntos. 


\section{DATOS Y ESTRATEGIA EMPÍRICA}

Para estudiar lo ocurrido con los beneficiados del puntaje ranking en términos de retención, utilizo datos administrativos provenientes del Departamento de Evaluación, Medición y Registro Educacional (Demre), de la Universidad de Chile, y del Servicio de Información de Educación Superior (SIES), del Ministerio de Educación. Estos registros incluyen información a nivel individual sobre los resultados de los estudiantes en la PSU, los programas a los que postulan y sus resultados en el proceso de admisión entre los años 2012 y 2016. Además, observo las ponderaciones utilizadas por las instituciones que participan del sistema centralizado de admisión para asignar sus vacantes y también la matrícula de educación superior, incluyendo la institución y programa al que asiste cada estudiante.

A partir de esta última información, construyo las variables dependientes que analizo: retención en el sistema, retención en la institución y retención en el programa tras el primer año de estudios. La retención a nivel de programa se refiere a estudiantes que tras el primer año siguen matriculados en el mismo programa e institución a los que ingresaron.

Además de documentar las tasas de retención de los estudiantes beneficiados por la inclusión del puntaje ranking, estudio también qué tan distintas son estas tasas de las observadas para otros grupos de estudiantes en el sistema. A través de estos análisis busco, por un lado, contextualizar su magnitud y, por otro, entregar ciertos elementos que contribuyan a la evaluación de la política. Aunque el foco de este trabajo son las tasas de retención al primer año, en el anexo 2 se presentan también análisis que estudian lo que ocurre al segundo año. ${ }^{15}$

Para implementar estos análisis utilizo simulaciones del proceso de selección. Las reglas de este proceso y las ponderaciones utilizadas para calcular los puntajes de los estudiantes son públicas. Esto, unido a la disponibilidad de los resultados de los procesos de admisión, hace posible replicar el algoritmo de asignación de vacantes y comprobar su

${ }^{15}$ Las diferencias en las tasas de retención de beneficiados y los otros grupos de estudiantes con los que se los compara aumentan o se mantienen en niveles similares a las documentadas para la retención al primer año. En el caso de las comparaciones con el grupo de perjudicados, esta diferencia aumenta. 
correcto funcionamiento. Para efectos de este proyecto, el algoritmo de selección fue programado en Python 2.7; su robustez se comprobó usando las postulaciones y resultados de quienes participaron en los procesos de admisión 2012 a 2015.

A partir de las simulaciones generadas con el algoritmo de selección, defino dos categorías de beneficiados y perjudicados: la primera, a nivel de sistema, y, la segunda, a nivel de programa.

El grupo de beneficiados a nivel de sistema incluye a aquellos estudiantes que fueron admitidos en alguna de las instituciones que participan del sistema único de admisión en los procesos 2013 a 2015, pero que no hubiesen sido seleccionados con las ponderaciones vigentes hasta el proceso de admisión 2012; es decir, estudiantes que sin el puntaje ranking hubiesen quedado fuera del sistema. La definición de los perjudicados, por otro otra parte, resulta más compleja; utilizar al grupo de estudiantes que deja de ingresar al sistema no es posible, ya que no observo sus tasas de retención. Por ello, defino al grupo de perjudicados como aquellos estudiantes que fueron admitidos en alguna de las instituciones del sistema en los procesos 2012 a 2014 pero que no hubiesen sido admitidos con las ponderaciones del año siguiente; es decir, estudiantes que no hubiesen sido admitidos en escenarios donde el puntaje ranking aumentó su relevancia (la ponderación promedio dada al puntaje ranking aumentó entre 2013 y 2015 de 10 a 23 por ciento).

Las definiciones de beneficiados y perjudicados a nivel de programa siguen una lógica similar. Aquellos estudiantes que en los procesos 2013 a 2015 fueron admitidos en una preferencia más alta que si hubiesen sido seleccionados con las ponderaciones del 2012 clasificaron como beneficiados. Aquellos estudiantes seleccionados en los procesos 2012 a 2014 que hubiesen sido admitidos en una preferencia más baja con las ponderaciones del año siguiente fueron clasificados como perjudicados.

Como puede apreciarse, los grupos de beneficiados son estudiantes que observo en el año t. Los perjudicados son estudiantes que observo en el año t-1. Idealmente, hubiese querido comparar las tasas de retención de estudiantes que ingresaron o dejaron de ingresar al sistema (o programa) al mismo tiempo. Desafortunadamente, no es posible observar lo que ocurre con quienes quedan fuera, y por ello, en los ejercicios en que comparo beneficiados y perjudicados, defino como grupo de 
control para los beneficiados del año $t$ a los perjudicados del año t-1; es decir, quienes fueron admitidos en ese período, pero que no hubiesen sido admitidos en el año t. Esta forma de comparar a beneficiados y perjudicados pudiese conducirnos a resultados sesgados si en el período estudiado hubiese cambios relevantes en las tasas de retención, inducidos, por ejemplo, por medidas pro retención de parte de las universidades. Sin embargo, como se aprecia en la tabla 1, éstas permanecen bastante estables.

Tabla 1. EVOLUCIÓN DE TASAS DE RETENCIÓN (2012-2015)

\begin{tabular}{lccc}
\hline & Sistema \% & Institución \% & Programa \% \\
\hline 2012 & 92,83 & 85,79 & 78,93 \\
2013 & 91,53 & 84,23 & 77,58 \\
2014 & 91,67 & 84,39 & 78,04 \\
2015 & 91,91 & 84,35 & 78,12 \\
\hline
\end{tabular}

Nota: Las cifras presentadas corresponden a las tasas de retención observadas en las instituciones que participan del sistema único de admisión.

Para comparar las tasas de retención de los beneficiados con otros grupos de estudiantes y también con los perjudicados utilizo regresiones del siguiente tipo:

$$
R_{i p t}=\beta_{p}+\beta_{t}+\beta_{1} B_{i p t}+\beta_{2} X_{i p t}+u_{i p t}
$$

donde:

$R_{i p t}$ : variable dummy que indica si el estudiante permanece en el sistema, en la institución o en el programa tras un año de estudios.

$B_{i p t}$ : variable dummy que indica si el estudiante pertenece al grupo de beneficiados.

$X_{i p t}$ : vector de controles a nivel individual que puede incluir características demográficas, socioeconómicas y académicas de los estudiantes.

$\beta_{1}$ : el coeficiente de interés, y captura la diferencia en las tasas de retención respecto a la categoría base, que, dependiendo de la especificación, definiré como el resto de los estudiantes matriculados en el sistema, aquellos matriculados que ingresaron en el 10 por ciento inferior de sus programas y el grupo de estudiantes perjudicados.

$\beta_{p}$ y $\beta_{t}$ : coeficientes que corresponden a efectos fijos a nivel de programa y efectos fijos por proceso. 
Por su naturaleza, los análisis discutidos hasta este punto asumen como dado lo que ocurre de manera previa a la selección de los estudiantes. Es decir, estos resultados deben ser interpretados con cautela si en respuesta a la introducción del puntaje ranking los estudiantes modificaran su conducta de alguna forma relevante en relación con sus probabilidades de admisión y retención. Algunas de estas potenciales respuestas incluyen: (i) incrementos en los niveles de esfuerzo de los estudiantes, (ii) transferencias estratégicas de escuela y (iii) cambios en las decisiones de postulación.

Si el puntaje ranking hubiese generado respuestas importantes de este tipo, entonces las definiciones de los grupos de beneficiados y perjudicados serían erróneas, afectando de este modo los resultados aquí discutidos.

Sin embargo, la posibilidad de responder, en cualquiera de estos márgenes, es inexistente o mucho menor en el primer año de la política. Ésta fue anunciada a mediados del año escolar (junio del 2012) y, ante la controversia que generó, su aplicación no fue confirmada de manera inmediata. En el anexo 1 se presentan análisis en los que se estudia exclusivamente a la primera cohorte afectada. Es alentador que los resultados de este ejercicio no difieran significativamente de los presentados en el cuerpo de este trabajo. ${ }^{16}$

Finalmente, cabe destacar que la mera existencia de cambios de conducta como los recién mencionados no es suficiente para afectar la validez de los análisis discutidos en este trabajo. Sólo debiesen preocuparnos en la medida en que cambien la composición de los grupos de beneficiados y perjudicados. ${ }^{17}$

${ }^{16}$ Respecto a los cambios en la estrategia de postulación, el hecho de que los estudiantes puedan postular hasta diez preferencias atenúa la preocupación por respuestas significativas en este margen. Menos del 6 por ciento de los estudiantes utiliza su décima preferencia con una postulación válida; de ellos, el 93 por ciento es seleccionado en una preferencia anterior. Dado que los cambios introducidos el primer año fueron menores a los incorporados posteriormente y que no existía información acerca de cómo cambiarían los puntajes de corte, respuestas de este tipo son aún menos plausibles en la primera cohorte afectada.

${ }^{17}$ Un elemento adicional a considerar es que por la forma en que construyeron los grupos de beneficiados y perjudicados éstos no pertenecen a la misma cohorte. El supuesto relevante en este punto es que las tasas de retención de los estudiantes perjudicados (seleccionados en t-1) son un buen reflejo de las tasas de retención que hubiesen tenido los estudiantes efectivamente perjudicados en el proceso de selección desarrollado en $\mathrm{t}$. 


\section{RESULTADOS}

En esta sección discuto los resultados obtenidos a partir de la estrategia descrita en la sección anterior. Parto por analizar las tasas de retención de los beneficiados en términos de acceso al sistema; es decir, las tasas de aquellos estudiantes que, sin el puntaje ranking, hubiesen quedado fuera de las universidades que participan del sistema único de admisión. Las características de este grupo de beneficiados y las del resto de los estudiantes que fueron admitidos en el sistema se presentan en la tabla 2. Esta tabla muestra que, en promedio, los beneficiados provienen de contextos más desaventajados que sus compañeros.

Es importante aclarar que esta diferencia no nos dice nada sobre cambios en la equidad del sistema. Para estudiar los efectos del puntaje ranking en equidad tendríamos que comparar al grupo de beneficiados con el grupo de estudiantes que dejó de ingresar debido a la incorporación de este instrumento en el mismo proceso de selección (véase Barrios 2017 para un análisis detallado sobre equidad en el acceso). Las diferencias aquí observadas son las que existen entre estudiantes que ingresan marginalmente al sistema y el resto de los estudiantes, que en promedio accedieron de manera más holgada.

Tras estudiar lo que ocurre a nivel del sistema, presento los resultados de un análisis similar, en el que me concentro únicamente en las dos instituciones más selectivas. En este caso, los alumnos beneficiados son aquellos que gracias al puntaje ranking lograron ingresar a alguna de estas dos instituciones.

Finalmente, presento resultados para los beneficiados en términos de programa; esto es, para aquellos estudiantes que gracias al puntaje ranking fueron admitidos en preferencias más altas. Sus características se presentan en la tabla 3. Aunque en esta ocasión las diferencias son algo menores, una vez más puede apreciarse que el grupo de beneficiados proviene en promedio de contextos más desaventajados. Por las mismas razones mencionadas anteriormente, estos resultados no nos dicen nada sobre cambios en la equidad del sistema. 
Tabla 2. ESTADÍSTICAS DESCRIPTIVAS DE BENEFICIADOS Y NO BENEFICIADOS (2013-2015) EN TÉRMINO DE SISTEMA

\begin{tabular}{|c|c|c|}
\hline & No beneficiados & Beneficiados \\
\hline \multicolumn{3}{|l|}{ 1. Caracteristicas demográficas } \\
\hline Sexo $=$ Femenino & 0.500 & 0.610 \\
\hline Edad & 18,682 & 18,766 \\
\hline \multicolumn{3}{|l|}{ 2. Seguro de salud } \\
\hline Fonasa & 0,536 & 0,704 \\
\hline Isapre & 0,388 & 0,219 \\
\hline Otro & 0,076 & 0.076 \\
\hline \multicolumn{3}{|c|}{ 3. Ingreso del hogar y educación de los padres } \\
\hline Ingreso bruto del hogar (\$ chilenos) & 802.403 & 516.699 \\
\hline Educación media incompleta & 0,098 & 0,171 \\
\hline Educación media completa & 0,305 & 0,422 \\
\hline Centro de formación técnica & 0,082 & 0,079 \\
\hline Instituto profesional & 0,082 & 0,083 \\
\hline Universidad & 0,391 & 0,223 \\
\hline \multicolumn{3}{|l|}{ 4. Ocupación de los padres } \\
\hline Activo(a) & 0,825 & 0,765 \\
\hline Parcialmente activo(a) & 0,074 & 0,102 \\
\hline No trabaja & 0,056 & 0,075 \\
\hline Otra situación ocupacional & 0,045 & 0,058 \\
\hline \multicolumn{3}{|l|}{ 5. Dependencia administrativa } \\
\hline Municipal & 0,224 & 0,284 \\
\hline Particular subvencionado & 0,516 & 0,594 \\
\hline Particular pagado & 0,242 & 0,086 \\
\hline Administración delegada & 0,017 & 0,037 \\
\hline
\end{tabular}

\begin{tabular}{lcc}
\hline 6. Nivel de selectividad institución educación superior & (IES) & \\
Altamente selectiva & 0,202 & 0,139 \\
Selectiva & 0,131 & 0,260 \\
Menor selectividad & 0,397 & 0,295 \\
No selectiva & 0,270 & 0,306 \\
\hline 7. Tasas de retención & 0,919 & 0,848 \\
Sistema & 0,845 & 0,774 \\
Institución & 0,781 & 0,713 \\
Programa & 223.088 & 6.372 \\
\hline Observaciones & & \\
\hline Nota: El grupo de beneficiados corresponde a aquellos alumnos que en ausencia del \\
puntaje ranking no hubiesen sido admitidos en el sistema.
\end{tabular}


Tabla 3. ESTADÍSTICAS DESCRIPTIVAS DE BENEFICIADOS Y NO BENEFICIADOS (2013-2015) EN TÉRMINOS DE PROGRAMA

\begin{tabular}{|c|c|c|}
\hline & No beneficiados & Beneficiados \\
\hline \multicolumn{3}{|l|}{ 1. Caracteristicas demográficas } \\
\hline Sexo = Femenino & 0,493 & 0,610 \\
\hline Edad & 18,693 & 18,588 \\
\hline \multicolumn{3}{|l|}{ 2. Seguro de salud } \\
\hline Fonasa & 0,528 & 0,675 \\
\hline Isapre & 0,396 & 0,252 \\
\hline Otro & 0,077 & 0,073 \\
\hline \multicolumn{3}{|c|}{ 3. Ingreso del hogar y educación de los padres } \\
\hline Ingreso bruto del hogar (\$ chilenos) & 814.976 & 579.137 \\
\hline Educación media incompleta & 0,095 & 0,162 \\
\hline Educación media completa & 0,300 & 0,392 \\
\hline Centro de formación técnica & 0,082 & 0,081 \\
\hline Instituto profesional & 0,083 & 0,076 \\
\hline Universidad & 0,398 & 0,263 \\
\hline \multicolumn{3}{|l|}{ 4. Ocupación de los padres } \\
\hline Activo(a) & 0,828 & 0,775 \\
\hline Parcialmente activo(a) & 0,072 & 0,101 \\
\hline No trabaja & 0,056 & 0,069 \\
\hline Otra situación ocupacional & 0,044 & 0,055 \\
\hline \multicolumn{3}{|l|}{ 5. Dependencia administrativa } \\
\hline Municipal & 0,221 & 0,280 \\
\hline Particular subvencionado & 0,513 & 0,573 \\
\hline Particular pagado & 0,249 & 0,114 \\
\hline Administración delegada & 0,016 & 0,033 \\
\hline \multicolumn{3}{|l|}{ 6. Nivel de selectividad IES } \\
\hline Altamente selectiva & 0,197 & 0,235 \\
\hline Selectiva & 0,124 & 0,250 \\
\hline Menor selectividad & 0,403 & 0,300 \\
\hline No selectiva & 0,276 & 0,215 \\
\hline \multicolumn{3}{|l|}{ 7. Tasas de retención } \\
\hline Sistema & 0,920 & 0,889 \\
\hline Institución & 0,846 & 0,812 \\
\hline Programa & 0,781 & 0,755 \\
\hline Observaciones & 209.507 & 19.953 \\
\hline
\end{tabular}

Nota: El grupo de beneficiados corresponde a aquellos alumnos que en ausencia del puntaje de ranking hubiesen sido admitidos en preferencias más bajas. 


\subsection{Retención y beneficiados en términos de acceso al sistema}

La tabla 4 estudia las diferencias en las tasas de retención tras el primer año entre los beneficiados y otros grupos de estudiantes. Las primeras dos columnas los compara con el resto de los estudiantes que ingresaron a las universidades que participan del sistema único de admisión junto a ellos; las siguientes dos con los estudiantes que ingresaron a sus programas en el diez por ciento inferior de los seleccionados, y las últimas dos, con quienes dejaron de entrar al sistema producto de la incorporación del ranking.

Los resultados presentados en el panel A muestran que las probabilidades de seguir matriculado en el sistema tras el primer año de estudios son 7 puntos porcentuales más bajas para los beneficiados que para el resto de sus compañeros. Al comparar las tasas de retención institucional y a nivel de programa para estos mismos grupos que se presentan en los paneles $\mathrm{B}$ y $\mathrm{C}$ de la misma tabla, también se observa una diferencia en contra de los estudiantes beneficiados cercana a los 8 puntos porcentuales.

La diferencia entre las dos columnas, que se presentan para cada grupo de comparación, está dada por los controles incluidos en cada especificación. En ambos casos se incluyen efectos fijos por programa de estudios y año. Incluir efectos fijos por programa es una manera de controlar por potenciales diferencias entre las carreras a las que ingresan los beneficiados y los estudiantes que forman parte del grupo de comparación. En la segunda columna se incluyen además controles demográficos y socioeconómicos. Sin embargo, no es del todo claro hasta qué punto es deseable controlar por estas variables en estas especificaciones.

Uno de los objetivos del puntaje de ranking consistía en mejorar la equidad en el acceso al sistema. Ello implica reemplazar a estudiantes provenientes de contextos más aventajados por estudiantes provenientes de contextos más desaventajados. Frente a ello, al controlar por características socioeconómicas estaríamos eliminando parte de las consecuencias de la política. Si los jóvenes que ingresaron al sistema (o a un programa) gracias al puntaje ranking fueran, en efecto, más vulnerables que quienes dejaron de hacerlo, y esta característica del grupo de beneficiados estuviese asociada a mayores dificultades para permanecer en la universidad, entonces incluir variables socioeconómicas al comparar 
Tabla 4. TASAS DE RETENCIÓN AL PRIMER AÑO: BENEFICIADOS A NIVEL DE SISTEMA VERSUS OTROS ESTUDIANTES (2013-2015)

\begin{tabular}{|c|c|c|c|c|c|c|}
\hline & \multicolumn{2}{|c|}{ Sistema } & \multicolumn{2}{|c|}{10 por ciento inferior } & \multicolumn{2}{|c|}{ Perjudicados } \\
\hline \multicolumn{7}{|c|}{ Panel A - Retención a nivel de sistema } \\
\hline \multirow[t]{2}{*}{ Beneficiado $=1$} & $-0,072^{* * *}$ & $-0,066^{* * *}$ & $-0,052^{\star * *}$ & $-0,046^{* * *}$ & $-0,026^{* *}$ & $-0,040^{* * *}$ \\
\hline & $(0,007)$ & $(0,005)$ & $(0,006)$ & $(0,006)$ & $(0,010)$ & $(0,012)$ \\
\hline \multirow[t]{2}{*}{ Constante } & $0,916^{* * *}$ & $0,917^{* * *}$ & $0,894^{* * *}$ & $0,791^{* * *}$ & $0,888^{\star \star *}$ & $1,045^{\star * *}$ \\
\hline & $(0,001)$ & $(0,018)$ & $(0,003)$ & $(0,208)$ & $(0,011)$ & $(0,040)$ \\
\hline
\end{tabular}

Panel B - Retención institucional

\begin{tabular}{lcccccc} 
Beneficiado=1 & $-0,079^{* * *}$ & $-0,076^{* * *}$ & $-0,055^{* * *}$ & $-0,051^{* * *}$ & $-0,024^{* *}$ & $-0,035^{* *}$ \\
\multirow{3}{*}{ Constante } & $(0,006)$ & $(0,006)$ & $(0,007)$ & $(0,007)$ & $(0,012)$ & $(0,014)$ \\
& $0,843^{* * *}$ & $0,951^{* *}$ & $0,815^{* * *}$ & $0,846^{* * *}$ & $0,820^{* * *}$ & $1,146^{* * *}$ \\
& $(0,003)$ & $(0,008)$ & $(0,004)$ & $(0,215)$ & $(0,012)$ & $(0,046)$ \\
\hline
\end{tabular}

Panel C - Retención a nivel de programa

\begin{tabular}{lcccccc} 
Beneficiado=1 & $-0,081^{* * *}$ & $-0,080^{* * *}$ & $-0,058^{* * *}$ & $-0,055^{* * *}$ & $-0,025^{*}$ & $-0,034^{* *}$ \\
& $(0,006)$ & $(0,006)$ & $(0,007)$ & $(0,008)$ & $(0,013)$ & $(0,015)$ \\
Constante & $0,779^{* * *}$ & $0,935^{* * *}$ & $0,752^{* * *}$ & $0,823^{* * *}$ & $0,746^{* * *}$ & $1,093^{* * *}$ \\
& $(0,006)$ & $(0,029)$ & $(0,005)$ & $(0,214)$ & $(0,014)$ & $(0,109)$ \\
\hline $\mathrm{N} .^{\circ}$ de estudiantes & 229.459 & 212.680 & 28.538 & 26.645 & 9.221 & 8.689 \\
\hline $\begin{array}{l}\text { Controles } \\
\text { demográficos }\end{array}$ & No & Si & No & Si & No & Si \\
$\begin{array}{l}\text { Controles } \\
\text { socioeconómicos }\end{array}$ & No & Si & No & Si & No & Si \\
$\begin{array}{l}\text { Efectos fijos por } \\
\text { programa }\end{array}$ & Si & Si & Si & Si & Si & Si \\
\hline
\end{tabular}

Notas: Los errores estándar son reportados en paréntesis. Corresponden a errores estándar robustos para cuyo cálculo se definieron clusters a nivel de programa de estudios: ${ }^{*}$ pvalue $<0,1,{ }^{* *} p$-value $<0,05,{ }^{* \star *} p$-value < 0,01. Los controles demográficos incluyen género y edad; los controles socioeconómicos incluyen dependencia escolar, ingresos brutos del hogar, educación y ocupación de los padres. Los estudiantes beneficiados corresponden a aquellos que ingresaron al sistema entre 2013 y 2015, pero que no hubiesen ingresado a él con las ponderaciones del 2012. Los estudiantes perjudicados son aquellos admitidos en el sistema entre 2012 y 2014, pero que no hubiesen sido seleccionados con las ponderaciones vigentes un año más tarde. 
a estos jóvenes con otros grupos de estudiantes eliminaría parte de las consecuencias generadas por la incorporación de este instrumento en el proceso de selección.

El incluir estas variables en las especificaciones estudiadas no genera grandes cambios en las diferencias estimadas. Esto sugiere que las diferencias en las tasas de retención aquí documentadas no se explican, en gran medida, por diferencias socioeconómicas de los estudiantes.

A pesar de contar con información sobre el rendimiento escolar de los estudiantes y los puntajes obtenidos en las distintas secciones de la PSU, no se presentan especificaciones que controlen por estas variables en el cuerpo del informe (en el anexo 3, publicado sólo en la version digital de este trabajo, pueden consultarse especificaciones alternativas). La razón es similar a la discutida en el párrafo anterior. Al incorporar el puntaje ranking al sistema de admisión, se modificaron las ponderaciones dadas a las distintas secciones de la PSU y a las notas de enseñanza media. Por lo tanto, uno de los efectos de la política es cambiar el perfil de puntajes de los seleccionados; como muestra Barrios (2017), los estudiantes beneficiados por el puntaje ranking tienen en promedio notas más altas y puntajes PSU más bajos que los perjudicados. Si mantenemos constantes estas variables al comparar a beneficiados con otros grupos de estudiantes se eliminaría esta parte de los efectos. ${ }^{18}$

Frente a ello, mi especificación preferida es la presentada en las columnas impares; es decir, la que controla únicamente por efectos fijos temporales y por programa. Esto aplica a todos los análisis presentados en este trabajo.

La comparación recién discutida no es necesariamente la más adecuada a la hora de evaluar esta política. En ella se contrastan unos estudiantes que marginalmente lograron ingresar al sistema ${ }^{19}$ con otros que en general consiguieron entrar con un margen mayor y que con o sin el puntaje ranking hubiesen ingresado al mismo programa. Por ello, en la tabla 4 también presento los resultados de un ejercicio similar, pero en los que el grupo de control está formado por aquellos estudiantes que

${ }^{18}$ En general, al controlar por estas variables, el tamaño del coeficiente que captura la diferencia entre beneficiados y otros estudiantes disminuye. Sin embargo, en la mayoría de los casos sigue habiendo una diferencia significativa.

${ }^{19}$ En promedio, los beneficiados se situaron en el 25 por ciento inferior de seleccionados; la mitad de ellos se situó en el 18 por ciento inferior de este grupo. 
ingresaron en el diez por ciento inferior de sus programas. Aun cuando en este caso la diferencia se reduce, siguen habiendo entre 4,5 y 6 puntos porcentuales de diferencia en todos los indicadores de retención; los beneficiados por el puntaje ranking presentan tasas de retención que se encuentran incluso por debajo del diez por ciento inferior de seleccionados (la diferencia varía entre 5 y 6 puntos porcentuales).

Hasta ahora sólo he comparado a los beneficiados con distintos grupos de estudiantes que ingresaron al sistema junto a ellos (es decir, estudiantes cuyo acceso a las universidades con el sistema único de admisión no se vio afectado por la incorporación del puntaje ranking). Las últimas columnas de la tabla 4 presentan los resultados de un análisis que estudia si el grupo de beneficiados presenta tasas de retención distintas a las que hubiesen tenido quienes quedaron fuera del sistema gracias a la inclusión del puntaje ranking. Una vez más, encuentro que el grupo de estudiantes beneficiados presenta tasas de retención menores a las del grupo de comparación; las tasas de retención a nivel de sistema, de instituciones y de programa son cerca de 2,5 puntos porcentuales menores para los beneficiados. Esto corresponde a tasas de deserción 23,21 por ciento mayores a nivel de sistema, 13,3 por ciento mayores a nivel de institución y 9,8 por ciento mayores a nivel de programa para beneficiados que para perjudicados.

De acuerdo a estos resultados, con la inclusión del puntaje de ranking se dejó fuera a un grupo de estudiantes cuyas tasas de retención hubiesen sido más altas.

Cabe destacar que este resultado no es necesariamente algo negativo. Si bien las tasas de retención del grupo de beneficiados son menores que las del resto de sus compañeros y que las del grupo de perjudicados, esto no quiere decir que ellas sean bajas. Una pérdida en esta dimensión pudiese ser algo aceptable a cambio de una mejora en equidad. Sin embargo, como se ha mencionado anteriormente, éste no parece ser el caso.

\subsection{Retención y beneficiados en términos de acceso a instituciones altamente selectivas}

La tabla 5 presenta los resultados de un ejercicio similar a los recién discutidos, pero en los que se incluyen únicamente a beneficiados y perjudicados en términos de acceso a las dos instituciones más se- 
Tabla 5. TASAS DE RETENCIÓN EN INSTITUCIONES ALTAMENTE SELECTIVAS: BENEFICIADOS VERSUS PERJUDICADOS (2013-2015)

\begin{tabular}{|c|c|c|}
\hline & M1 & M2 \\
\hline \multicolumn{3}{|c|}{ Panel A - Retención a nivel de sistema } \\
\hline \multirow[t]{2}{*}{ Beneficiado=1 } & 0,012 & 0,005 \\
\hline & $(0,010)$ & $(0,011)$ \\
\hline \multirow[t]{2}{*}{ Constante } & $0,957^{* * *}$ & $-0,089$ \\
\hline & $(0,009)$ & $(0,067)$ \\
\hline \multicolumn{3}{|c|}{ Panel B - Retención institucional } \\
\hline \multirow[t]{2}{*}{ Beneficiado $=1$} & $-0,034$ & $-0,059^{*}$ \\
\hline & $(0,031)$ & $(0,031)$ \\
\hline \multirow[t]{2}{*}{ Constante } & $0,842^{* * *}$ & $-0,029$ \\
\hline & $(0,017)$ & $(0,096)$ \\
\hline \multicolumn{3}{|c|}{ Panel C - Retención a nivel de programa } \\
\hline \multirow[t]{2}{*}{ Beneficiado=1 } & $-0,032$ & $-0,051^{*}$ \\
\hline & $(0,029)$ & $(0,030)$ \\
\hline \multirow[t]{2}{*}{ Constante } & $0,777^{* * *}$ & 0,035 \\
\hline & $(0,024)$ & $(0,121)$ \\
\hline N..$^{\circ}$ de estudiantes & 1.729 & 1.556 \\
\hline Controles demográficos & No & Si \\
\hline Controles socioeconómicos & No & Si \\
\hline Efectos fijos por programa & No & Sí \\
\hline Efectos fijos por año & Si & Sí \\
\hline
\end{tabular}

Notas: Los errores estándar son reportados en paréntesis. Corresponden a errores estándar robustos para cuyo cálculo se definieron clusters a nivel de programa de estudios: ${ }^{*} p$-value < 0,1, ${ }^{* *} p$-value < 0,05, ${ }^{* * *} p$-value < 0,01. Los controles demográficos incluyen género y edad; los controles socioeconómicos incluyen dependencia escolar, ingresos brutos del hogar, educación y ocupación de los padres. Los estudiantes beneficiados corresponden a aquellos que ingresaron a universidades altamente selectivas entre 2013 y 2015 , pero que no hubiesen ingresado a ellas con las ponderaciones del 2012. Los estudiantes perjudicados son aquellos admitidos a estas instituciones entre 2012 y 2014, pero que no hubiesen sido seleccionados con las ponderaciones vigentes un año más tarde. 
lectivas del sistema. Con este análisis, busco estudiar cómo varían los resultados discutidos en la sección anterior con el nivel de selectividad de las instituciones.

En términos de permanencia en el sistema, no se observan diferencias significativas entre el grupo de beneficiados y perjudicados. Esto puede deberse, en parte, a que los admitidos en instituciones altamente selectivas, antes de abandonar el sistema, tienen la posibilidad de moverse a múltiples instituciones con menores niveles de selectividad.

Al estudiar lo que ocurre a nivel institucional y de programa, mi especificación preferida indica que las tasas de retención a nivel institucional y de programa son cerca de tres puntos porcentuales menores que las de los estudiantes perjudicados. A pesar de que estas diferencias son mayores que las observadas para el resto del sistema, no son estadísticamente significativas. En parte, esto se debe al tamaño de la muestra; en este caso, es un quinto de la analizada en la sección anterior y los errores estándar son tres veces mayores.

Una vez más, cabe destacar que aunque las tasas de retención del grupo de beneficiados son menores que las de los estudiantes perjudicados, éstas no son necesariamente bajas; tanto a nivel institucional como a nivel de programa, las tasas de retención de este grupo superan el 70 por ciento.

\subsection{Retención y beneficiados en términos de acceso a programas}

Hasta ahora, los análisis presentados han estado enfocados en beneficiados a nivel de sistema; es decir, en estudiantes que fueron admitidos en algún programa de las instituciones que participan del sistema único de admisión gracias a la incorporación del puntaje ranking.

En esta sección estudiaré lo ocurrido con otro grupo de beneficiados: aquellos estudiantes que gracias a la incorporación del puntaje ranking fueron admitidos en programas que se encontraban más arriba en su lista de preferencias.

Para ser consistente con la definición utilizada para los beneficiados, el grupo de perjudicados estará compuesto por estudiantes que con el puntaje ranking (o una mayor ponderación de este instrumento) hubiesen sido admitidos en preferencias más bajas que en las que fueron admitidos el año en que los observo. De manera similar a lo realizado 
al analizar al grupo de beneficiados a nivel de sistema, al comparar a beneficiados y perjudicados a nivel de preferencias, compararé a estudiantes que ingresaron al sistema en $\mathrm{t}$ y $\mathrm{t}-1$.

La tabla 6 presenta los resultados de comparaciones entre este grupo de beneficiados y (1) el resto de sus compañeros en el sistema, (2) el diez por ciento inferior de seleccionados en cada programa y (3) quienes fueron perjudicados por el puntaje ranking (es decir, quienes en escenarios en que el puntaje ranking tiene mayor importancia hubiesen sido seleccionados en preferencias menores). Las comparaciones con estos tres grupos de estudiantes muestran que las tasas de retención de los beneficiados son menores. En el primer caso, las diferencias a nivel de sistema, de institución y de programa varían entre cinco y seis puntos porcentuales.

La comparación con el diez por ciento inferior de seleccionados arroja resultados bastante similares. Se observan diferencias de entre 3,5 y 4,5 puntos porcentuales en las distintas tasas de retención.

Finalmente, la comparación entre beneficiados y perjudicados muestra que es menos probable que un estudiante beneficiado permanezca en la misma institución y programa en que se matriculó inicialmente. Si estos números se expresan en términos de tasas de deserción, las del grupo de beneficiados son 29,11 por ciento mayores a nivel de sistema, 14,83 por ciento mayores a nivel institucional y 9,5 por ciento a nivel de programa.

Una vez más, estos resultados no quieren decir que las tasas de retención de beneficiados sean bajas: tanto a nivel institucional, como de programa, superan el 70 por ciento. El tamaño de la diferencia debe ser juzgado en relación con las ganancias generadas en términos de equidad. Además, las instituciones pueden afectar estas diferencias. A cambio de mayor equidad — algo que, en este caso, no es del todo claro que se logre-, las universidades podrían invertir recursos adicionales para apoyar la integración y adecuado desempeño de estudiantes provenientes de contextos más desaventajados.

Más allá de las posibles vías de acción que tienen las instituciones, lo que muestran los análisis discutidos en esta sección es que la calidad de los estudiantes beneficiados, medida en términos de retención al primer año, no es mayor que la del grupo de estudiantes perjudicados. De hecho, los resultados indican justamente lo contrario. 
Tabla 6. TASAS DE RETENCIÓN AL PRIMER AÑO: BENEFICIADOS A NIVEL DE PROGRAMA VERSUS OTROS ESTUDIANTES (2013-2015)

\begin{tabular}{|c|c|c|c|c|c|c|}
\hline & \multicolumn{2}{|c|}{ Sistema } & \multicolumn{2}{|c|}{$10 \%$ inferior } & \multicolumn{2}{|c|}{ Perjudicados } \\
\hline \multicolumn{7}{|c|}{ Panel A - Retención a nivel de sistema } \\
\hline \multirow[t]{2}{*}{ Beneficiado=1 } & $-0,051^{\star \star \star}$ & $-0,048^{* * *}$ & $-0,036^{\star * *}$ & $-0,033^{* * *}$ & $-0,023^{* * *}$ & $-0,039^{* \star *}$ \\
\hline & $(0,003)$ & $(0,003)$ & $(0,004)$ & $(0,004)$ & $(0,004)$ & $(0,006)$ \\
\hline \multirow[t]{2}{*}{ Constante } & $0,918^{* * *}$ & $0,944^{* * *}$ & $0,911^{* * *}$ & $0,933^{* * *}$ & $0,921^{* * *}$ & $0,975^{* * *}$ \\
\hline & $(0,001)$ & $(0,005)$ & $(0,003)$ & $(0,018)$ & $(0,004)$ & $(0,015)$ \\
\hline \multicolumn{7}{|c|}{ Panel B - Retención institucional } \\
\hline \multirow[t]{2}{*}{ Beneficiado=1 } & $-0,060^{\star * *}$ & $-0,061^{\star * *}$ & $-0,041^{\star \star \star}$ & $-0,044^{* * *}$ & $-0,023^{* * *}$ & $-0,041^{* \star *}$ \\
\hline & $(0,004)$ & $(0,004)$ & $(0,005)$ & $(0,005)$ & $(0,006)$ & $(0,007)$ \\
\hline \multirow[t]{2}{*}{ Constante } & $0,846^{* * *}$ & $0,846^{* * *}$ & $0,835^{* * *}$ & $1,032^{* * *}$ & $0,845^{* * *}$ & $1,075^{\star * *}$ \\
\hline & $(0,001)$ & $(0,001)$ & $(0,004)$ & $(0,017)$ & $(0,006)$ & $(0,020)$ \\
\hline \multicolumn{7}{|c|}{ Panel C - Retención a nivel de programa } \\
\hline \multirow[t]{2}{*}{ Beneficiado $=1$} & $-0,062^{\star * *}$ & $-0,064^{* * *}$ & $-0,044^{* * *}$ & $-0,044^{* * *}$ & $-0,021^{* * *}$ & $-0,037^{* * *}$ \\
\hline & $(0,004)$ & $(0,004)$ & $(0,005)$ & $(0,006)$ & $(0,007)$ & $(0,008)$ \\
\hline \multirow[t]{2}{*}{ Constante } & $0,781^{* * *}$ & $0,971^{\star \star *}$ & $0,776^{* * *}$ & $0,978^{* * *}$ & $0,778^{* * *}$ & $1,006^{* \star *}$ \\
\hline & $(0,003)$ & $(0,009)$ & $(0,005)$ & $(0,018)$ & $(0,008)$ & $(0,022)$ \\
\hline N..$^{\circ}$ de estudiantes & 229.459 & 212.680 & 37.372 & 34.921 & 30.712 & 28.699 \\
\hline $\begin{array}{l}\text { Controles } \\
\text { demográficos }\end{array}$ & No & Si & No & Sí & No & Sí \\
\hline $\begin{array}{l}\text { Controles } \\
\text { socioeconómicos }\end{array}$ & No & Si & No & Si & No & Si \\
\hline $\begin{array}{l}\text { Efectos fijos por } \\
\text { programa }\end{array}$ & Si & Si & $\mathrm{Si}$ & Si & $\mathrm{Si}$ & Si \\
\hline Efectos fijos por año & Si & Sí & Si & Si & Si & Si \\
\hline
\end{tabular}

Notas: Los errores estándar son reportados en paréntesis. Corresponden a errores estándar robustos para cuyo cálculo se definieron clusters a nivel de programa de estudios: ${ }^{*} p$-value $<0,1,{ }^{* *} p$-value < 0,05, ${ }^{* \star *} p$-value < 0,01. Los controles demográficos incluyen género y edad; los controles socioeconómicos incluyen dependencia escolar, ingresos brutos del hogar, educación y ocupación de los padres, Los estudiantes beneficiados corresponden a aquellos que entre 2013 y 2015 fueron admitidos en una preferencia mayor que en la que hubiesen sido aceptados con las ponderaciones del año 2012. Los estudiantes perjudicados corresponden a quienes entre 2012 y 2014 fueron admitidos en una preferencia mayor que la que hubiesen sido seleccionados un año después. 


\section{CONCLUSIONES}

En 2012, el Consejo de Rectores de las Universidades Chilenas aprobó la inclusión del puntaje ranking en el sistema de admisión universitario. A través de esta medida, buscaba reconocer el mérito académico de los estudiantes en su contexto escolar y, con ello, disminuir los niveles de desigualdad en el sistema, sin reducir la calidad de los estudiantes seleccionados.

En este trabajo estudio las tasas de retención de quienes fueron beneficiados por esta medida y las comparo con las de otros grupos de estudiantes, incluyendo a quienes fueron perjudicados por ella. Con este fin, simulé los resultados del proceso de admisión y, a partir de ellos, definí dos categorías de estudiantes beneficiados y dos de estudiantes perjudicados. La primera, en términos de acceso al sistema, y, la segunda, en términos de la preferencia en que fueron admitidos.

Al comparar las tasas de retención tras el primer año, tanto a nivel de sistema como a nivel institucional y de programa, encuentro que las tasas de retención de quienes accedieron a alguna de las instituciones que participan del sistema único de admisión, gracias a la inclusión del puntaje de ranking, son entre 5 y 8 puntos porcentuales menores que las del resto de sus compañeros y entre 2,5 y 3,5 puntos porcentuales menores que las de los estudiantes perjudicados. Estas últimas diferencias corresponden a tasas de deserción 23,21 por ciento mayores a nivel de sistema, 13,3 por ciento mayores a nivel de institución y 9,8 por ciento mayores a nivel de programa.

$\mathrm{Al}$ analizar lo que ocurre exclusivamente en las dos instituciones más selectivas del sistema, encuentro resultados muy similares. Una vez más, los estudiantes beneficiados presentan tasas de retención institucional, y, a nivel de programa, menores que las de los perjudicados. Las diferencias encontradas superan marginalmente los tres puntos porcentuales.

Las tasas de retención de la segunda categoría de beneficiados, es decir, de aquellos estudiantes que gracias al puntaje ranking fueron admitidos en preferencias más altas, son una vez más menores que las de sus compañeros, incluyendo a aquellos seleccionados en el diez por ciento inferior de sus programas. Sus tasas de retención son también menores que las de los estudiantes perjudicados. En términos de retención en el sistema, y, a nivel institucional y de programa, la diferencia fluctúa entre 2,1 y 2,3 puntos porcentuales. En términos de tasas de deserción, las del grupo de beneficiados son 29,11 por ciento mayores 
a nivel de sistema, 14,83 por ciento mayores a nivel institucional y 9,5 por ciento mayores a nivel de programa.

A la hora de seleccionar estudiantes, pudiese ser aceptable reemplazar a un grupo de postulantes por otros individuos con menores tasas de retención y rendimiento académico, si esto viniese acompañado de avances en equidad. Aunque los resultados de Barrios (2017) muestran que esta política no generó grandes cambios en esa dimensión, el juicio sobre la conveniencia de su adopción es de carácter normativo. Para algunos, una pequeña ganancia en equidad pudiese justificar pérdidas en términos de retención.

Cabe destacar que aunque las tasas de retención de ambos grupos de beneficiados son menores que las observadas para los estudiantes perjudicados, éstas no son necesariamente bajas. En todos los casos estudiados siempre se encuentran por sobre el 70 por ciento.

Es importante mencionar, además, que estas tasas no son inamovibles, ya que las instituciones pueden tomar medidas para modificarlas. A cambio de una mayor equidad - algo que no es del todo claro que se haya logrado con la política aquí estudiada-, las universidades podrían invertir recursos adicionales para apoyar la integración y adecuado desempeño de estudiantes provenientes de contextos más desaventajados.

Algo que dificulta este tipo de iniciativas pro retención es que por la forma en que funciona el sistema de admisión resulta difícil identificar al grupo de estudiantes beneficiados por el puntaje ranking. Esto, a su vez, dificulta el diseño de programas focalizados en este grupo de estudiantes. Desde esa perspectiva, sería interesante poder generar indicadores que les permitan a las instituciones identificar a estudiantes en mayor riesgo de deserción para prestarles el apoyo que requieran.

Estando ya en su sexto año de funcionamiento esta medida, se extrañan más estudios sobre su funcionamiento y la discusión de otras alternativas que pudiesen contribuir a avanzar de manera más significativa en términos de equidad.

Para evitar que en el futuro ocurra algo similar con otras reformas al sistema de admisión, sería bueno establecer con anterioridad a la implementación de reformas los criterios bajo los cuales se evaluará su éxito. Además, para facilitar esta tarea será importante incorporar también en su diseño una metodología de evaluación adecuada; los problemas para identificar de manera precisa los efectos del puntaje ranking en los distintos outcomes de interés y juzgar su éxito tienen su origen, precisamente, en la omisión de estos elementos en su diseño original. 


\section{REFERENCIAS}

Antonovics, K. \& B. Backes. 2014. "The Effect of Banning Affirmative Action on College Admission Policies and Student Quality". Journal of Human Resources 49 (2): 295-322.

Arcidiacono, P., E. Aucejo, P. Coate \& J. Hotz. 2014. "Affirmative Action and University Fit: Evidence from Proposition 209". IZA Journal of Labor Economics 3 (1): 2487-2529.

Arcidiacono, P., E. Aucejo \& K. Spenner. 2012. "What Happens After Enrollment? An Analysis of the Time Path of Racial Differences in GPA and Major Choice". IZA Journal of Labor Economics 1 (5).

Barrios, A. 2017. "Combatiendo la desigualdad en el acceso a la universidad: El caso del puntaje de ranking". Presentación en seminario "Efectos del ranking de notas en la admisión a las universidades chilenas". Centro de Estudios Públicos, 12 de diciembre de 2017.

Cortes, K. E. 2010. "Do Bans on Affirmative Action Hurt Minority Students? Evidence from the Texas top 10 \% Plan". Economics of Education Review 29 (6): 1110-1124.

Cruch. 2017. "Estudio acerca de la validez predictiva del ranking de notas". Consejo de Rectores de las Universidades Chilenas.

Gil, F. J., R. Paredes \& I. Sánchez. 2013. "El ranking de las notas: inclusión con excelencia”. Temas de la Agenda Pública 8 (60). Centro de Políticas Públicas $\mathrm{UC}$.

Kapor, A. 2016. "Distributional Effects of Race-blind Affirmative Action". Manuscrito.

Koljatic, M. y M. Silva. 2013. "Opening a Side-gate: Engaging the Excluded in Chilean Higher Education through Test-blind Admission". Studies in Higher Education 38: 1427-1441.

Lance, L. 2011. "Nonproduction Benefits of Education". Handbook of the Economics of Education 4: 183-282.

Larrocau, T., I. Ríos \& A. Mizala. 2013. "Efecto de la incorporación del ranking de notas en la selección universitaria”. Departamento de Evaluación, Medición y Registros Académicos. Universidad de Chile.

Oded, G. 2011. "Inequality, Human Capital Formation, and the Process of Development". Handbook of the Economics of Education 4: 441-493.

Santalices, V., X. Catalán, C. Horn \& A. Venegas. 2017. "High School Rankings in University Admissions at National Level: Theory of Action and Early Results from Chile". Higher Education Policy: 1-21. 


\section{ANEXO 1. Tasas de retención beneficiados 2013}

Tabla 7. TASAS DE RETENCIÓN: BENEFICIADOS A NIVEL DE SISTEMA VERSUS OTROS ESTUDIANTES (2013)

\begin{tabular}{lccc}
\hline & Sistema & $10 \%$ inferior & Perjudicados \\
\hline Panel A - Retención a nivel de sistema & & & \\
Beneficiado=1 & $-0,052^{* * *}$ & $-0,034^{* * *}$ & $-0,028$ \\
& $(0,011)$ & $(0,014)$ & $(0,028)$ \\
Constante & $0,916^{* * *}$ & $0,892^{* * *}$ & $0,884^{* * *}$ \\
& $(0,000)$ & $(0,002)$ & $(0,015)$ \\
\hline Panel B - Retención institucional & & & \\
Beneficiado=1 & $-0,052^{* * *}$ & $-0,031^{*}$ & $-0,026^{* * *}$ \\
& $(0,013)$ & $(0,016)$ & $(0,034)$ \\
Constante & $0,843^{* * *}$ & $0,810^{* * *}$ & $0,811^{* * *}$ \\
& $(0,000)$ & $(0,002)$ & $(0,018)$ \\
\hline Panel C - Retención a nivel de programa & & & \\
Beneficiado=1 & $-0,060^{* * *}$ & $-0,037^{* * *}$ & $-0,036$ \\
& $(0,014)$ & $(0,017)$ & $(0,038)$ \\
Constante & $0,777^{* * *}$ & $0,746^{* * *}$ & $0,733^{* * *}$ \\
& $(0,000)$ & $(0,002)$ & $(0,021)$ \\
\hline N. de estudiantes & 77.212 & 8.637 & 1.913 \\
\hline Efectos fijos por programa & Si & Si & Si \\
Efectos fijos por año & Si & Si & Si \\
\hline
\end{tabular}

Notas: Los errores estándar son reportados en paréntesis. Corresponden a errores estándar robustos para cuyo cálculo se definieron clusters a nivel de programa de estudios: ${ }^{*} p$-value $<0,1,{ }^{* *} p$-value $<0,05,{ }^{* * *} p$-value < 0,01. Los estudiantes beneficiados corresponden a aquellos que ingresaron al sistema el 2013, pero que no hubiesen ingresado al sistema con las ponderaciones vigentes el 2012. Los estudiantes perjudicados son aquellos que ingresaron al sistema el 2012, pero que no lo hubiesen hecho con las ponderaciones vigentes un año más tarde. 
Tabla 8. TASAS DE RETENCIÓN: BENEFICIADOS A NIVEL DE PROGRAMA VERSUS OTROS ESTUDIANTES (2013)

\begin{tabular}{lccc}
\hline & Sistema & $10 \%$ inferior & Perjudicados \\
\hline Panel A - Retención a nivel de sistema & & & \\
Beneficiado $=1$ & $-0,031^{* * *}$ & $-0,015^{*}$ & $-0,017^{* *}$ \\
& $(0,005)$ & $(0,008)$ & $(0,012)$ \\
Constante & $0,917^{* * *}$ & $0,900^{* * *}$ & $0,916^{* * *}$ \\
& $(0,000)$ & $(0,003)$ & $(0,005)$ \\
\hline Panel B - Retención institucional & & & \\
Beneficiado=1 & $-0,040^{* * *}$ & $-0,021^{* *}$ & $-0,035^{* * *}$ \\
& $(0,007)$ & $(0,011)$ & $(0,014)$ \\
Constante & $0,845^{* * *}$ & $0,820^{* * *}$ & $0,845^{* * *}$ \\
& $(0,000)$ & $(0,004)$ & $(0,006)$ \\
\hline Panel C - Retención a nivel de programa & & & \\
Beneficiado=1 & $-0,039^{* * *}$ & $-0,024^{* *}$ & $-0,035^{* * *}$ \\
& $(0,007)$ & $(0,011)$ & $(0,013)$ \\
Constante & $0,778^{* * *}$ & $0,760^{* * *}$ & $0,766^{* * *}$ \\
\hline Ef. de estudiantes & $(0,000)$ & $(0,004)$ & $(0,007)$ \\
\hline Efectos fijos por programa & 77.212 & 10.400 & 7.977 \\
\hline
\end{tabular}

Notas: Los errores estándar son reportados en paréntesis. Corresponden a errores estándar robustos para cuyo cálculo se definieron clusters a nivel de programa de estudios: ${ }^{*} p$-value <0,1, ${ }^{* *} p$-value <0,05, ${ }^{* * *} p$-value < 0,01. Los estudiantes beneficiados corresponden a aquellos que entre 2013 y 2015 fueron admitidos en una preferencia mayor que en la que hubiesen sido aceptados con las ponderaciones del año 2012. Los estudiantes perjudicados corresponden a quienes entre 2012 y 2014 fueron admitidos en una preferencia mayor que la que hubiesen sido seleccionados un año después. 


\section{ANEXO 2. Tasas de retención al segundo año}

Tabla 9. TASAS DE RETENCIÓN AL SEGUNDO AÑO: BENEFICIADOS A NIVEL DE SISTEMA VERSUS OTROS ESTUDIANTES (2013-2014)

\begin{tabular}{lccc}
\hline & Sistema & $10 \%$ inferior & Perjudicados \\
\hline Panel A - Retención a nivel de sistema & & & \\
Beneficiado=1 & $-0,091^{* * *}$ & $-0,050^{* * *}$ & $-0,054^{* * *}$ \\
& $(0,007)$ & $(0,009)$ & $(0,013)$ \\
Constante & $0,867^{* * *}$ & $0,825^{* * *}$ & $0,838^{* * *}$ \\
& $(0,001)$ & $(0,003)$ & $(0,013)$ \\
\hline Panel B - Retención institucional & & & \\
Beneficiado=1 & $-0,102^{* * *}$ & $-0,052^{* * *}$ & $-0,046^{* * *}$ \\
& $(0,008)$ & $(0,010)$ & $(0,016)$ \\
Constante & $0,759^{* * *}$ & $0,703^{* * *}$ & $0,725^{* * *}$ \\
& $(0,001)$ & $(0,004)$ & $(0,014)$ \\
\hline Panel C - Retención a nivel de programa & & & \\
Beneficiado=1 & $-0,095^{* * *}$ & $-0,051^{* * *}$ & $-0,046^{* *}$ \\
& $(0,009)$ & $(0,011)$ & $(0,017)$ \\
Constante & $0,659^{* * *}$ & $0,612^{* * *}$ & $0,631^{* * *}$ \\
& $(0,002)$ & $(0,004)$ & $(0,014)$ \\
\hline N. de estudiantes & 154.215 & 18.340 & 5.914 \\
\hline Efectos fijos por programa & Si & Si & Si \\
Efectos fijos por año & Si & Si & Si \\
\hline
\end{tabular}

Notas: Los errores estándar son reportados en paréntesis. Corresponden a errores estándar robustos para cuyo cálculo se definieron clusters a nivel de programa de estudios: ${ }^{*} p$-value $<0,1,{ }^{* *} p$-value $<0,05,{ }^{* \star *} p$-value $<0,01$. Los estudiantes beneficiados corresponden a aquellos que ingresaron al sistema entre 2013 y 2014, pero que no hubiesen ingresado a él con las ponderaciones del 2012. Los estudiantes perjudicados son aquellos admitidos en el sistema entre 2012 y 2013 , pero que no hubiesen sido seleccionados con las ponderaciones vigentes un año más tarde. 
Tabla 10. TASAS DE RETENCIÓN AL SEGUNDO AÑO: BENEFICIADOS A NIVEL DE PROGRAMA VERSUS OTROS ESTUDIANTES (2013-2014)

\begin{tabular}{|c|c|c|c|}
\hline & Sistema & $10 \%$ inferior & Perjudicados \\
\hline \multicolumn{4}{|c|}{ Panel A - Retención a nivel de sistema } \\
\hline \multirow[t]{2}{*}{ Beneficiado=1 } & $-0,064^{* * *}$ & $-0,033^{* * *}$ & $-0,031^{* * *}$ \\
\hline & $(0,004)$ & $(0,006)$ & $(0,005)$ \\
\hline \multirow[t]{2}{*}{ Constante } & $0,868^{* * *}$ & $0,848^{* \star *}$ & $0,870^{* * *}$ \\
\hline & $(0,003)$ & $(0,004)$ & $(0,005)$ \\
\hline \multicolumn{4}{|c|}{ Panel B - Retención institucional } \\
\hline \multirow[t]{2}{*}{ Beneficiado=1 } & $-0,080^{* * *}$ & $-0,035^{\star \star *}$ & $-0,032^{* * *}$ \\
\hline & $(0,005)$ & $(0,008)$ & $(0,007)$ \\
\hline \multirow[t]{2}{*}{ Constante } & $0,762^{* * *}$ & $0,727^{* * *}$ & $0,755^{\star * *}$ \\
\hline & $(0,001)$ & $(0,005)$ & $(0,006)$ \\
\hline \multicolumn{4}{|c|}{ Panel C - Retención a nivel de programa } \\
\hline \multirow[t]{2}{*}{ Beneficiado=1 } & $-0,079^{\star * *}$ & $-0,035^{* * *}$ & $-0,040^{* * *}$ \\
\hline & $(0,005)$ & $(0,008)$ & $(0,008)$ \\
\hline \multirow[t]{2}{*}{ Constante } & $0,663^{* * *}$ & $0,639^{* * *}$ & $0,673^{* * *}$ \\
\hline & $(0,002)$ & $(0,005)$ & $(0,007)$ \\
\hline $\mathrm{N} .^{\circ}$ de estudiantes & 154.215 & 23.523 & 21.330 \\
\hline Efectos fijos por programa & Si & Sí & Sí \\
\hline Efectos fijos por año & Si & Si & Si \\
\hline
\end{tabular}

Notas: Los errores estándar son reportados en paréntesis. Corresponden a errores estándar robustos para cuyo cálculo se definieron clusters a nivel de programa de estudios: ${ }^{*} p$-value $<0,1,{ }^{* *} p$-value $<0,05,{ }^{* * *} p$-value < 0,01. Los estudiantes beneficiados corresponden a aquellos que entre 2013 y 2014 fueron admitidos en una preferencia mayor que en la que hubiesen sido aceptados con las ponderaciones del año 2012. Los estudiantes perjudicados corresponden a quienes entre 2012 y 2013 fueron admitidos en una preferencia mayor que la que hubiesen sido seleccionados un año después. 
ANEXO 3. Tasas de retención - otras especificaciones

Tabla 11. TASAS DE RETENCIÓN AL PRIMER AÑO: BENEFICIADOS A NIVEL DE SISTEMA VERSUS RESTO DE LOS SELECCIONADOS (2013-2015)

(1) (2) (3)

(4)

\begin{tabular}{lcccc}
\hline Panel A - Retención a nivel de sistema & & & & \\
Beneficiado=1 & $-0,071^{* * *}$ & $-0,072^{* * *}$ & $-0,066^{* * *}$ & $-0,049^{* * *}$ \\
& $(0,005)$ & $(0,005)$ & $(0,005)$ & $(0,005)$ \\
Constante & $0,916^{* * *}$ & $0,916^{* * *}$ & $0,917^{* * *}$ & $0,596^{* * *}$ \\
& $(0,002)$ & $(0,001)$ & $(0,018)$ & $(0,024)$ \\
\hline Panel B - Retención institucional & & & & \\
Beneficiado=1 & $-0,072^{* * *}$ & $-0,079^{* * *}$ & $-0,076^{* * *}$ & $-0,055^{* * *}$ \\
& $(0,006)$ & $(0,006)$ & $(0,006)$ & $(0,006)$ \\
Constante & $0,843^{* * *}$ & $0,844^{* * *}$ & $0,951^{* * *}$ & $0,528^{* * *}$ \\
& $(0,003)$ & $(0,001)$ & $(0,008)$ & $(0,020)$ \\
\hline Panel C - Retención a nivel de programa & & & & \\
Beneficiado=1 & $-0,069^{* * *}$ & $-0,081^{* * *}$ & $-0,080^{* * *}$ & $-0,058^{* * *}$ \\
& $(0,009)$ & $(0,006)$ & $(0,006)$ & $(0,006)$ \\
Constante & $0,777^{* * *}$ & $0,779^{* * *}$ & $0,935^{* * *}$ & $0,495^{* * *}$ \\
& $(0,006)$ & $(0,003)$ & $(0,029)$ & $(0,037)$ \\
\hline N. de estudiantes & 229.459 & 229.459 & 212.680 & 212.680 \\
\hline Controles demográficos & No & No & Si & Si \\
Controles socioeconómicos & No & No & Si & Si \\
Controles académicos & No & No & No & Si \\
Efectos fijos por programa & No & Si & Si & Si \\
\hline & & Si \\
\hline
\end{tabular}

Notas: Los errores estándar son reportados en paréntesis. Corresponden a errores estándar robustos para cuyo cálculo se definieron clusters a nivel de programa de estudios: ${ }^{*} p$-value $<0,1,{ }^{* *} p$-value $<0,05,{ }^{* * *} p$-value $<0,01$. Los controles demográficos incluyen género y edad; los controles socioeconómicos incluyen dependencia escolar, ingresos brutos del hogar, educación y ocupación de los padres; finalmente, los controles académicos incluyen notas de enseñanza media y puntajes PSU. Los estudiantes beneficiados corresponden a aquellos que ingresaron al sistema entre 2013 y 2015, pero que no hubiesen accedido al sistema con las ponderaciones del 2012. 
Tabla 12. TASAS DE RETENCIÓN AL PRIMER AÑO: BENEFICIADOS A NIVEL DE SISTEMA VERSUS 10\% INFERIOR DE LOS SELECCIONADOS (2013-2015)

\begin{tabular}{lcccc}
\hline & $(1)$ & $(2)$ & $(3)$ & $(4)$ \\
\hline Panel A - Retención a nivel de sistema & & & & \\
Beneficiado=1 & $-0,047^{* * *}$ & $-0,052^{* * *}$ & $-0,046^{* * *}$ & $-0,037^{* * *}$ \\
& $(0,006)$ & $(0,006)$ & $(0,006)$ & $(0,006)$ \\
Constante & $0,894^{* * *}$ & $0,894^{* * *}$ & $0,791^{* * *}$ & $0,630^{* * *}$ \\
& $(0,004)$ & $(0,003)$ & $(0,208)$ & $(0,213)$ \\
\hline Panel B - Retención institucional & & & & \\
Beneficiado=1 & $-0,037^{* * *}$ & $-0,055^{* * *}$ & $-0,051^{* * *}$ & $-0,040^{* * *}$ \\
& $(0,007)$ & $(0,007)$ & $(0,007)$ & $(0,007)$ \\
Constante & $0,811^{* * *}$ & $0,815^{* * *}$ & $0,846^{* * *}$ & $0,730^{* * *}$ \\
& $(0,006)$ & $(0,004)$ & $(0,215)$ & $(0,224)$ \\
\hline Panel C - Retención a nivel de programa & & & & \\
Beneficiado=1 & $-0,033^{* * *}$ & $-0,058^{* * *}$ & $-0,055^{* * *}$ & $-0,044^{* * *}$ \\
& $(0,010)$ & $(0,007)$ & $(0,008)$ & $(0,008)$ \\
Constante & $0,745^{* * *}$ & $0,752^{* * *}$ & $0,823^{* * *}$ & $0,769^{* * *}$ \\
& $(0,007)$ & $(0,005)$ & $(0,214)$ & $(0,224)$ \\
\hline Ef. de estudiantes & 28.538 & 28.538 & 26.645 & 26.645 \\
\hline Controles demográficos & No & No & Si & Si \\
Controles socioeconómicos & No & No & Si & Si \\
\hline Nontroles académicos & No & No & No & Si \\
No & Si & Si & Si & Si \\
\hline
\end{tabular}

Notas: Los errores estándar son reportados en paréntesis. Corresponden a errores estándar robustos para cuyo cálculo se definieron clusters a nivel de programa de estudios: ${ }^{*} p$-value $<0,1,{ }^{* *} p$-value $<0,05,{ }^{* * *} p$-value $<0,01$. Los controles demográficos incluyen género y edad; los controles socioeconómicos incluyen dependencia escolar, ingresos brutos del hogar, educación y ocupación de los padres; finalmente, los controles académicos incluyen notas de enseñanza media y puntajes PSU. Los estudiantes beneficiados corresponden a aquellos que ingresaron al sistema entre 2013 y 2015, pero que no hubiesen accedido al sistema con las ponderaciones del 2012. 
Tabla 13. TASAS DE RETENCIÓN AL PRIMER AÑO: BENEFICIADOS A NIVEL DE SISTEMA VERSUS PERJUDICADOS (2013-2015)

\begin{tabular}{|c|c|c|c|c|}
\hline & (1) & (2) & (3) & (4) \\
\hline \multicolumn{5}{|c|}{ Panel A - Retención a nivel de sistema } \\
\hline \multirow[t]{2}{*}{ Beneficiado $=1$} & $-0,008$ & $-0,026^{* *}$ & $-0,040^{* * *}$ & $-0,028^{* *}$ \\
\hline & $(0,009)$ & $(0,010)$ & $(0,012)$ & $(0,014)$ \\
\hline \multirow[t]{2}{*}{ Constante } & $0,873^{\star \star \star}$ & $0,888^{\star \star *}$ & $1,045^{\star * *}$ & $0,802^{\star \star *}$ \\
\hline & $(0,010)$ & $(0,011)$ & $(0,040)$ & $(0,092)$ \\
\hline \multicolumn{5}{|c|}{ Panel B - Retención institucional } \\
\hline \multirow[t]{2}{*}{ Beneficiado=1 } & $-0,008$ & $-0,024^{* *}$ & $-0,035^{* *}$ & $-0,022$ \\
\hline & $(0,010)$ & $(0,012)$ & $(0,014)$ & $(0,015)$ \\
\hline \multirow[t]{2}{*}{ Constante } & $0,801^{* * *}$ & $0,820^{* * *}$ & $1,146^{* * *}$ & $0,927^{* * *}$ \\
\hline & $(0,012)$ & $(0,012)$ & $(0,046)$ & $(0,107)$ \\
\hline \multicolumn{5}{|c|}{ Panel C - Retención a nivel de programa } \\
\hline \multirow[t]{2}{*}{ Beneficiado $=1$} & 0,009 & $-0,025^{*}$ & $-0,034^{* *}$ & $-0,016$ \\
\hline & $(0,012)$ & $(0,013)$ & $(0,015)$ & $(0,016)$ \\
\hline \multirow[t]{2}{*}{ Constante } & $0,709^{* * *}$ & $0,746^{* * *}$ & $1,093^{* *}$ & $0,972^{* * *}$ \\
\hline & $(0,016)$ & $(0,014)$ & $(0,051)$ & $(0,109)$ \\
\hline $\mathrm{N} .^{\circ}$ de estudiantes & 9.221 & 9.221 & 8.689 & 8.689 \\
\hline Controles demográficos & No & No & Si & Sí \\
\hline Controles socioeconómicos & No & No & Si & Si \\
\hline Controles académicos & No & No & No & Si \\
\hline Efectos fijos por programa & No & Si & Si & Sí \\
\hline Efectos fijos por año & Si & $\mathrm{Si}$ & Si & Si \\
\hline
\end{tabular}

Notas: Los errores estándar son reportados en paréntesis. Corresponden a errores estándar robustos para cuyo cálculo se definieron clusters a nivel de programa de estudios: ${ }^{*} p$-value $<0,1,{ }^{* *} p$-value $<0,05,{ }^{* * *} p$-value $<0,01$. Los controles demográficos incluyen género y edad; los controles socioeconómicos incluyen dependencia escolar, ingresos brutos del hogar, educación y ocupación de los padres; finalmente, los controles académicos incluyen notas de enseñanza media y puntajes PSU. Los estudiantes beneficiados corresponden a aquellos que ingresaron al sistema entre 2013 y 2015, pero que no hubiesen accedido al sistema con las ponderaciones del 2012. Los estudiantes perjudicados son aquellos admitidos en el sistema entre 2012 y 2014, pero que no hubiesen sido seleccionados con las ponderaciones vigentes un año más tarde. 
Tabla 14. TASAS DE RETENCIÓN EN INSTITUCIONES ALTAMENTE SELECTIVAS: BENEFICIADOS VERSUS PERJUDICADOS (2013-2015)

\begin{tabular}{|c|c|c|c|c|}
\hline & (1) & (2) & (3) & (4) \\
\hline \multicolumn{5}{|c|}{ Panel A - Retención a nivel de sistema } \\
\hline \multirow[t]{2}{*}{ Beneficiado=1 } & 0,009 & 0,012 & 0,005 & 0,005 \\
\hline & $(0,010)$ & $(0,010)$ & $(0,011)$ & $(0,011)$ \\
\hline \multirow[t]{2}{*}{ Constante } & $0,957^{* * *}$ & $0,957^{* * *}$ & $-0,089$ & 0,082 \\
\hline & $(0,011)$ & $(0,009)$ & $(0,067)$ & $(0,126)$ \\
\hline \multicolumn{5}{|c|}{ Panel B - Retención institucional } \\
\hline \multirow[t]{2}{*}{ Beneficiado $=1$} & $-0,028$ & $-0,034$ & $-0,059^{*}$ & $-0,064^{* *}$ \\
\hline & $(0,028)$ & $(0,031)$ & $(0,031)$ & $(0,031)$ \\
\hline \multirow[t]{2}{*}{ Constante } & $0,837^{* * *}$ & $0,842^{* \star *}$ & $-0,029$ & 0,097 \\
\hline & $(0,021)$ & $(0,017)$ & $(0,096)$ & $(0,185)$ \\
\hline \multicolumn{5}{|c|}{ Panel C - Retención a nivel de programa } \\
\hline \multirow[t]{2}{*}{ Beneficiado $=1$} & $-0,026$ & $-0,032$ & $-0,051^{*}$ & $-0,053^{*}$ \\
\hline & $(0,027)$ & $(0,029)$ & $(0,030)$ & $(0,029)$ \\
\hline \multirow[t]{2}{*}{ Constante } & $0,776^{* * *}$ & $0,777^{* \star *}$ & 0,035 & $-0,080$ \\
\hline & $(0,026)$ & $(0,024)$ & $(0,121)$ & $(0,254)$ \\
\hline $\mathrm{N} .^{\circ}$ de estudiantes & 1.729 & 1.729 & 1.556 & 1.556 \\
\hline Controles demográficos & No & No & Si & Si \\
\hline Controles socioeconómicos & No & No & Si & Si \\
\hline Controles académicos & No & No & No & Si \\
\hline Efectos fijos por programa & No & Si & Si & Si \\
\hline Efectos fijos por año & $\mathrm{Si}$ & Sí & Si & Si \\
\hline
\end{tabular}

Notas: Los errores estándar son reportados en paréntesis. Corresponden a errores estándar robustos para cuyo cálculo se definieron clusters a nivel de programa de estudios: ${ }^{*} p$-value $<0,1,{ }^{* *} p$-value $<0,05,{ }^{* * *} p$-value $<0,01$. Los controles demográficos incluyen género y edad; los controles socioeconómicos incluyen dependencia escolar, ingresos brutos del hogar, educación y ocupación de los padres; finalmente, los controles académicos incluyen notas de enseñanza media y puntajes PSU. Los estudiantes beneficiados corresponden a aquellos que ingresaron a universidades altamente selectivas entre 2013 y 2015, pero que no hubiesen accedido a ellas con las ponderaciones de 2012. Los estudiantes perjudicados son aquellos admitidos a estas instituciones entre 2012 y 2014, pero que no hubiesen sido seleccionados con las ponderaciones vigentes un año más tarde. 
Tabla 15. TASAS DE RETENCIÓN AL PRIMER AÑO: BENEFICIADOS A NIVEL DE PROGRAMA VERSUS RESTO DE LOS SELECCIONADOS (2013-2015)
(1)
(2)
(3)
(4)

\begin{tabular}{|c|c|c|c|c|}
\hline \multirow[t]{2}{*}{ Beneficiado $=1$} & $-0,032^{* * *}$ & $-0,051^{\star * *}$ & $-0,048^{* * *}$ & $-0,032^{* * *}$ \\
\hline & $(0,004)$ & $(0,003)$ & $(0,003)$ & $(0,003)$ \\
\hline \multirow[t]{2}{*}{ Constante } & $0,917^{* * *}$ & $0,918^{* * *}$ & $0,944^{* * *}$ & $0,623^{* * *}$ \\
\hline & $(0,002)$ & $(0,001)$ & $(0,005)$ & $(0,017)$ \\
\hline \multicolumn{5}{|c|}{ Panel B - Retención institucional } \\
\hline \multirow[t]{2}{*}{ Beneficiado=1 } & $-0,034^{\star \star *}$ & $-0,060^{* * *}$ & $-0,061^{* * *}$ & $-0,041^{\star \star *}$ \\
\hline & $(0,004)$ & $(0,004)$ & $(0,004)$ & $(0,004)$ \\
\hline \multirow[t]{2}{*}{ Constante } & $0,844^{\star \star *}$ & $0,846^{\star \star *}$ & $0,985^{* * *}$ & $0,565^{\star * *}$ \\
\hline & $(0,003)$ & $(0,001)$ & $(0,017)$ & $(0,020)$ \\
\hline \multicolumn{5}{|c|}{ Panel C - Retención a nivel de programa } \\
\hline \multirow[t]{2}{*}{ Beneficiado=1 } & $-0,027^{\star * *}$ & $-0,062^{* * *}$ & $-0,064^{* * *}$ & $-0,043^{* * *}$ \\
\hline & $(0,006)$ & $(0,004)$ & $(0,004)$ & $(0,004)$ \\
\hline \multirow[t]{2}{*}{ Constante } & $0,777^{\star \star \star}$ & $0,781^{\star \star \star}$ & $0,971^{* * \star}$ & $0,535^{\star \star \star}$ \\
\hline & $(0,006)$ & $(0,003)$ & $(0,009)$ & $(0,027)$ \\
\hline N. ${ }^{\circ}$ de estudiantes & 229.459 & 229.459 & 212.680 & 212.680 \\
\hline Controles demográficos & No & No & Si & Si \\
\hline Controles socioeconómicos & No & No & Si & Si \\
\hline Controles académicos & No & No & No & $\mathrm{Si}$ \\
\hline Efectos fijos por programa & No & Si & Si & Sí \\
\hline Efectos fijos por año & Si & Si & Si & Si \\
\hline
\end{tabular}

Notas: Los errores estándar son reportados en paréntesis. Corresponden a errores estándar robustos para cuyo cálculo se definieron clusters a nivel de programa de estudios: ${ }^{*} p$-value $<0,1,{ }^{* *} p$-value $<0,05,{ }^{* * *} p$-value $<0,01$. Los controles demográficos incluyen género y edad; los controles socioeconómicos incluyen dependencia escolar, ingresos brutos del hogar, educación y ocupación de los padres; finalmente, los controles académicos incluyen notas de enseñanza media y puntajes PSU. Los estudiantes beneficiados corresponden a aquellos que entre 2013 y 2015 fueron admitidos en una preferencia mayor que en la que hubiesen sido seleccionados con las ponderaciones del año 2012 . 
Tabla 16. TASAS DE RETENCIÓN AL PRIMER AÑO: BENEFICIADOS A NIVEL DE PROGRAMA VERSUS 10\% INFERIOR DE LOS SELECCIONADOS (2013-2015)

\begin{tabular}{|c|c|c|c|c|}
\hline & (1) & (2) & (3) & (4) \\
\hline \multicolumn{5}{|c|}{ Panel A - Retención a nivel de sistema } \\
\hline \multirow[t]{2}{*}{ Beneficiado=1 } & $-0,002$ & $-0,036^{* * *}$ & $-0,033^{* * *}$ & $-0,021^{\star \star *}$ \\
\hline & $(0,004)$ & $(0,004)$ & $(0,004)$ & $(0,005)$ \\
\hline \multirow[t]{2}{*}{ Constante } & $0,894^{* * *}$ & $0,911^{* * *}$ & $0,933^{* * *}$ & $0,707^{* * *}$ \\
\hline & $(0,004)$ & $(0,003)$ & $(0,013)$ & $(0,047)$ \\
\hline \multicolumn{5}{|c|}{ Panel B - Retención institucional } \\
\hline \multirow[t]{2}{*}{ Beneficiado=1 } & 0,005 & $-0,041^{* * *}$ & $-0,044^{* * *}$ & $-0,031^{* \star *}$ \\
\hline & $(0,006)$ & $(0,005)$ & $(0,005)$ & $(0,006)$ \\
\hline \multirow[t]{2}{*}{ Constante } & $0,809^{\star * *}$ & $0,835^{\star \star *}$ & $1,032^{* \star *}$ & $0,795^{\star \star \star}$ \\
\hline & $(0,006)$ & $(0,004)$ & $(0,017)$ & $(0,057)$ \\
\hline \multicolumn{5}{|c|}{ Panel C - Retención a nivel de programa } \\
\hline \multirow[t]{2}{*}{ Beneficiado $=1$} & $0,015^{\star *}$ & $-0,044^{* * *}$ & $-0,047^{\star * *}$ & $-0,035^{* * *}$ \\
\hline & $(0,007)$ & $(0,005)$ & $(0,006)$ & $(0,006)$ \\
\hline \multirow[t]{2}{*}{ Constante } & $0,743^{* * *}$ & $0,776^{* * *}$ & $0,978^{* * *}$ & $0,782^{* * *}$ \\
\hline & $(0,007)$ & $(0,005)$ & $(0,018)$ & $(0,059)$ \\
\hline $\mathrm{N} .^{\circ}$ de estudiantes & 37.372 & 37.372 & 34.921 & 34.921 \\
\hline Controles demográficos & No & No & Si & Si \\
\hline Controles socioeconómicos & No & No & Si & Sí \\
\hline Controles académicos & No & No & No & Si \\
\hline Efectos fijos por programa & No & Si & Si & Si \\
\hline Efectos fijos por año & Si & Si & Si & Si \\
\hline
\end{tabular}

Notas: Los errores estándar son reportados en paréntesis. Corresponden a errores estándar robustos para cuyo cálculo se definieron clusters a nivel de programa de estudios: ${ }^{*} p$-value $<0,1,{ }^{* *} p$-value $<0,05,{ }^{* * *} p$-value $<0,01$. Los controles demográficos incluyen género y edad; los controles socioeconómicos incluyen dependencia escolar, ingresos brutos del hogar, educación y ocupación de los padres; finalmente, los controles académicos incluyen notas de enseñanza media y puntajes PSU. Los estudiantes beneficiados corresponden a aquellos que entre 2013 y 2015 fueron admitidos en una preferencia mayor que en la que hubiesen sido aceptados con las ponderaciones del año 2012 . 
Tabla 17. TASAS DE RETENCIÓN AL PRIMER AÑO: BENEFICIADOS A NIVEL DE PROGRAMA VERSUS PERJUDICADOS (2013-2015)
(1)
(2)
(3)
(4)

\begin{tabular}{lcccc}
\hline Panel A - Retención a nivel de sistema & & & & \\
Beneficiado=1 & $-0,007$ & $-0,023^{* * *}$ & $-0,039^{* * *}$ & $-0,024^{* * *}$ \\
& $(0,005)$ & $(0,004)$ & $(0,006)$ & $(0,006)$ \\
Constante & $0,911^{* * *}$ & $0,921^{* * *}$ & $0,975^{* * *}$ & $0,791^{* * *}$ \\
& $(0,005)$ & $(0,004)$ & $(0,015)$ & $(0,046)$ \\
\hline Panel B - Retención institucional & & & & \\
Beneficiado=1 & 0,001 & $-0,023^{* * *}$ & $-0,041^{* * *}$ & $-0,021^{* * *}$ \\
& $(0,006)$ & $(0,006)$ & $(0,007)$ & $(0,007)$ \\
Constante & $0,825^{* * *}$ & $0,845^{* * *}$ & $1,075^{* * *}$ & $0,860^{* * *}$ \\
\hline & $(0,007)$ & $(0,006)$ & $(0,020)$ & $(0,058)$ \\
\hline Panel C - Retención a nivel de programa & & & & \\
Beneficiado=1 & $0,020^{* *}$ & $-0,021^{* * *}$ & $-0,037^{* * *}$ & $-0,014$ \\
& $(0,009)$ & $(0,007)$ & $(0,008)$ & $(0,009)$ \\
Constante & $0,736^{* * *}$ & $0,778^{* * *}$ & $1,006^{* * *}$ & $0,835^{* * *}$ \\
& $(0,014)$ & $(0,008)$ & $(0,022)$ & $(0,062)$ \\
\hline N. ${ }^{\circ}$ de estudiantes & 30,712 & 30.712 & 28.699 & 28.699 \\
\hline Controles demográficos & No & No & Si & Si \\
Controles socioeconómicos & No & No & Si & Si \\
Controles académicos & No & No & No & Si \\
Efectos fijos por programa & No & Si & Si & Si \\
Efectos fijos por año & Si & Si & Si \\
\hline
\end{tabular}

Notas: Los errores estándar son reportados en paréntesis. Corresponden a errores estándar robustos para cuyo cálculo se definieron clusters a nivel de programa de estudios: ${ }^{*} p$-value $<0,1,{ }^{* *} p$-value $<0,05,{ }^{* * *} p$-value $<0,01$. Los controles demográficos incluyen género y edad; los controles socioeconómicos incluyen dependencia escolar, ingresos brutos del hogar, educación y ocupación de los padres; finalmente, los controles académicos incluyen notas de enseñanza media y puntajes PSU. Los estudiantes beneficiados corresponden a aquellos que entre 2013 y 2015 fueron admitidos en una preferencia mayor que en la que hubiesen sido seleccionados con las ponderaciones del año 2012. Los estudiantes perjudicados corresponden a quienes entre 2012 y 2014 fueron admitidos en una preferencia mayor que la que hubiesen sido aceptados un año después. 